\title{
OGLE-2014-BLG-1186: gravitational microlensing providing evidence for a planet orbiting the foreground star or for a close binary source?
}

\author{
M. Dominik ${ }^{\oplus},{ }^{\dagger}$ E. Bachelet,,${ }^{2,3}$ V. Bozza,,${ }^{4,5}$ R. A. Street,${ }^{2}$ C. Han,${ }^{6}$ \\ M. Hundertmark, ${ }^{1,7,8}$ A. Udalski, ${ }^{9}$ D. M. Bramich, ${ }^{10}$ K. A. Alsubai, ${ }^{3}$ S. Calchi Novati, ${ }^{11}$ \\ S. Ciceri, ${ }^{12}$ G. D’Ago ${ }^{\oplus},{ }^{4,13,14,15}$ R. Figuera Jaimes, ${ }^{1,2,16,17}$ T. Haugbølle, ${ }^{7}$ \\ T. C. Hinse, ${ }^{18,19}$ K. Horne, ${ }^{1}$ U. G. Jørgensen, ${ }^{7}$ D. Juncher, ${ }^{7}$ N. Kains ${ }^{\oplus},{ }^{20}$ \\ H. Korhonen ${ }^{\oplus}{ }^{21}$ L. Mancini, ${ }^{12,22,23}$ J. Menzies ${ }^{\oplus},{ }^{24}$ A. Popovas ${ }^{\oplus},{ }^{7}$ M. Rabus ${ }^{\circledR},{ }^{12,15}$ \\ S. Rahvar ${ }^{\oplus},{ }^{25}$ G. Scarpetta, ${ }^{4,13}$ R. Schmidt, ${ }^{8}$ J. Skottfelt, ${ }^{7,26}$ C. Snodgrass ${ }^{\circledR},{ }^{27,28,29}$ \\ J. Southworth, ${ }^{30}$ D. Starkey, ${ }^{1}$ I. A. Steele, ${ }^{31}$ J. Surdej, ${ }^{32}$ Y. Tsapras,,${ }^{2,8,33}$ \\ J. Wambsganss, ${ }^{8,34}$ O. Wertz, ${ }^{32}$ P. Pietrukowicz, ${ }^{9}$ M. K. Szymański, ${ }^{9}$ P. Mróz, ${ }^{9}$ \\ J. Skowron ${ }^{\oplus},{ }^{9}$ I. Soszyński, ${ }^{9}$ K. Ulaczyk, ${ }^{9,35}$ R. Poleski, ${ }^{9,36}$ Ł. Wyrzykowski ${ }^{9}$ \\ and S. Kozłowski ${ }^{9}$
}

Affiliations are listed at the end of the paper

Accepted 2019 January 24. Received 2019 January 11; in original form 2018 August 8

\begin{abstract}
Discussing the particularly long gravitational microlensing event OGLE-2014-BLG-1186 with a time-scale $t_{\mathrm{E}} \sim 300 \mathrm{~d}$, we present a methodology for identifying the nature of localised deviations from single-lens point-source light curves, which ensures that (1) the claimed signal is substantially above the noise floor, (2) the inferred properties are robustly determined and their estimation is not subject to confusion with systematic noise in the photometry, (3) alternative viable solutions within the model framework are not missed. Annual parallax and binarity could be separated and robustly measured from the wing and the peak data, respectively. We find matching model light curves that involve either a binary lens or a binary source, and discover hitherto unknown model ambiguities. Our binary-lens models indicate a planet of mass $M_{2}=(45 \pm 9) \mathrm{M}_{\oplus}$, orbiting a star of mass $M_{1}=(0.35 \pm 0.06) \mathrm{M}_{\odot}$, located at a distance $D_{\mathrm{L}}=(1.7 \pm 0.3) \mathrm{kpc}$ from Earth, whereas our binary-source models suggest a brown-dwarf lens of $M=(0.046 \pm 0.007) \mathrm{M}_{\odot}$, located at a distance $D_{\mathrm{L}}=(5.7 \pm 0.9) \mathrm{kpc}$, with the source potentially being a (partially) eclipsing binary involving stars predicted to be of similar colour given the ratios between the luminosities and radii. Further observations might resolve the ambiguity in the interpretation in favour of either a lens or a source binary. We experienced that close binary source stars pose a challenge for claiming the detection of planets by microlensing in events where the source passes very close to the lens star hosting the planet.
\end{abstract}

Key words: gravitational lensing: micro-methods: data analysis-methods: statisticalplanets and satellites: detection-(stars:) binaries: eclipsing-Galaxy: kinematics and dynamics.
^E-mail: md35@st-andrews.ac.uk

$\dagger$ Royal Society University Research Fellow.

\section{INTRODUCTION}

The vast majority of claimed microlensing planet detections are based on a pretty obvious signal in the acquired photometric data (e.g. Bond et al. 2004; Udalski et al. 2005; Beaulieu et al. 2006; Sumi et al. 2010; Gaudi et al. 2008; Muraki et al. 2011). This 
makes one wonder why detections from less obvious signals (e.g. Dong et al. 2009; Janczak et al. 2010) are scarce, given that more subtle features should be quite common. Clearly, if more subtle features are discarded altogether, we lose out on the significance of the planet population statistics arising from the acquired data, and we lose sensitivity particularly to low-mass companions. Moreover, sampling events more densely than necessary can be quite a waste of telescope resources, and strongly diminish the overall detection efficiency of follow-up campaigns (e.g. Dominik et al. 2002, 2007, 2010; Horne, Snodgrass \& Tsapras 2009; Tsapras et al. 2009). The detection efficiency (e.g. Gaudi \& Sackett 2000; Rhie et al. 2000) is a crucial characteristic, with planets probabilistically escaping their detection through microlensing even with perfectly sampled and precise photometric light curves (Mao \& Paczyński 1991), depending on where they happen to be located along their orbit during the course of a microlensing event.

If we assume a photometric time-series composed of $N$ data points $\left(t_{i}, F_{i}, \sigma_{i}\right)$ with measured fluxes $F_{i}$ and estimated uncertainties $\sigma_{i}$, as well as a theoretical light curve $F\left(t_{i}\right)$, one finds the sum of the squared standardised residuals as

$\chi^{2} \equiv \sum_{i=1}^{N}\left(\frac{F_{i}-F\left(t_{i}\right)}{\sigma_{i}}\right)^{2}$.

As compared to gravitational microlensing by a single isolated lens star (Einstein 1936; Paczyński 1986), a quasi-static binarylens system (e.g. a star with a single planet) is characterised by an additional three parameters (Mao \& Paczyński 1991). Moreover, a planetary signature also usually reveals the angular size of the source star, described by a further parameter. For such a signature, one therefore finds only a small probability $\mathcal{P}_{4}\left(\Delta \chi^{2} \geq 20\right)=4 \times 10^{-4}$ for a difference in $\chi^{2}$ in excess of 20 for 4 additional degrees of freedom. This means that a likelihood ratio test suggests a clear signal for e.g. as few as 5 data points at the $2 \sigma$ level, under the provision that the measurement uncertainties are accurately estimated, uncorrelated, and follow a Gaussian profile.

However, in reality it cannot be tacitly assumed that these conditions hold, and we rather need to be careful about false positives lurking in the actual noise of the photometric measurements. Even a high detection threshold does not provide an insurance policy on this because correlated noise (or 'red noise') can lead to 'pseudodetections' at arbitrarily large $\Delta \chi^{2}$ if just the cadence of the photometric time-series is high enough. In fact, in at least one case, the careful analysis of an observed gravitational microlensing event arrived at the conclusion that a putative planetary signal is likely due to red noise (Bachelet et al. 2015).

A consistent interpretation of data requires to demonstrate that putative signals are not likely to arise from noise, and adequate criteria are required to distinguish signals from the noise floor. It would be obviously inconsistent to claim a detection of a signal from data that show deviations that are similar to what is being considered 'noise' for other data. It is therefore indicated to establish a suitable 'noise' model and estimate some 'noise' statistics.

Blind searches in high-dimensional non-linear parameter spaces bear a substantial risk of confusing true signals in the data with noise. It is rather straightforward to find a good match between noise patterns and models describing small localised deviations, as previous analyses of microlensing events explicitly demonstrated (e.g. Bozza et al. 2012).

Signals of low-mass planets and satellites may be subtle, but fortunately these are well localised. In other words, the vast majority of photometric data provide no relevant constraint to the model parameters that describe the anomaly. Moreover, all the other parameters can usually be well determined from the data not containing the anomaly. This permits splitting up parameter space into two subspaces with disjoint associated data sets. Looking at the effect of the anomaly region on the anomaly-independent parameters provides a valuable consistency check, while the data not covering the putative anomaly can be used to infer parameters describing noise statistics that do not depend on any assumptions about the anomaly. It should however be noted that while such an approach works well for weak anomaly features, strong features (e.g. due to caustic passages) can be highly sensitive to the track of the source relative to the lens system, thereby substantially affecting a large number of model parameters.

In this article, we discuss the microlensing event OGLE-2014BLG-1186, which not only is of exceptionally long duration but also shows a putative anomaly in the form of a close double peak. We explicitly demonstrate how this anomaly can be systematically and robustly identified and present viable interpretations of its physical nature. Gravitational microlensing events that show a photometric light curve involving two peaks can result from either (or both) a lens binary (Mao \& Paczyński 1991; Gould \& Loeb 1992; Griest \& Safizadeh 1998) or a source binary (Griest \& Hu 1992). Gaudi (1998) discussed an ambiguity between planetary binary-lens and binary-source models for putative planetary signatures that arise from the source passing close to one of the 'planetary caustics' (see Section 3.3.1), so that the light ray passes close to the planet (Erdl \& Schneider 1993). In the case of OGLE-2014-BLG-1186, we are however facing a different situation, where the source passes close to the central caustic of the putative binary-lens system, located near the position of the planet's host star.

In Section 2, we describe our data acquisition and original identification of a putative anomaly over the peak of the light curve, while Section 3 is devoted to a detailed account of our modelling efforts. We discuss the physical nature of the lens and source objects and the wider significance of our findings in Section 4. We draw final conclusions in Section 5.

\section{DATA ACQUISITION}

\subsection{Survey and follow-up}

Soon after Mao \& Paczyński (1991) demonstrated that the gravitational microlensing effect could be used to detect extra-solar planets, Gould \& Loeb (1992) argued that a combination of survey and follow-up would be an efficient way to do so. With the implementation of the 'Early Warning System' (EWS; Udalski et al. 1994) by the Optical Gravitational Lensing Experiment (OGLE) team, the real-time detection of microlensing events became public information, enabling a wider scientific community to engage in harvesting the scientific returns of these transient phenomena.

In 2014, the fourth phase of OGLE (OGLE-IV; Udalski, Szymański \& Szymański 2015) was in operation, using the $1.3 \mathrm{~m}$ Warsaw University Telescope at Las Campanas Observatory in Chile and a mosaic camera of 32 E2V44-82 $2048 \times 4102$ CCD chips with $I$ - and $V$-band filters, delivering a total field of view of $1.4 \mathrm{deg}^{2}$ at $0.26 \mathrm{arcsec}$ pixel $^{-1}{ }^{1}$. The current implementation of the OGLE-IV EWS, using a photometric data pipeline based on Difference Image Analysis (DIA) photometry (Alard \& Lupton 1998; Alard 2000; Woźniak 2000), assesses about 380 million stars

\footnotetext{
${ }^{1}$ http://ogle.astrouw.edu.pl/main/OGLEIV/mosaic.html
} 
in 85 Galactic bulge fields, leading to 2049 microlensing events announced in 2014.

\subsection{The RoboNet campaign}

The RoboNet microlensing campaign makes use of the Las Cumbres Observatory (LCO) network ${ }^{2}$ of globally distributed $1 \mathrm{~m}$ and $2 \mathrm{~m}$ telescopes, operated by LCOGT Inc. (Goleta, California). Three of the southern $1 \mathrm{~m}$ telescopes are owned by the University of St Andrews, which in turn holds a respective fraction of observing time on the network. LCO's $1 \mathrm{~m}$ telescopes are organised in clusters at four sites in the network. Due to the location of the Galactic bulge, we are using only the three telescopes at the Cerro-Tololo Interamerican Observatory (CTIO, Chile), the three at the South African Astronomical Observatory (SAAO, South Africa), and two installed alongside LCO's 2 m telescope (Faulkes Telescope South, FTS) at the Siding Spring Observatory (SSO, Australia).

All of the telescopes are robotically operated. At the time of these observations, most $1 \mathrm{~m}$ telescopes hosted SBIG STX-16803 cameras with Kodak KAF-16803 front illuminated $4096 \times 4096$ pix CCDs. These instruments have a field of view of $15.8 \mathrm{arcmin}^{2}$ and a pixel scale of 0.464 arcsec pixel $^{-1}$ when used in the standard bin $2 \times 2$ mode. Two $1 \mathrm{~m}$ telescopes in Chile supported Sinistro cameras, which consist of $4096 \times 4096$ pixel Fairchild CCD486 back-illuminated CCDs operated in bin $1 \times 1$ mode to produce a $26.5 \mathrm{arcmin}^{2}$ field with a pixel scale of $0.387 \mathrm{arcsec} \mathrm{pixel}^{-1}$. The $1 \mathrm{~m}$ telescopes are designed to be as identical as possible to facilitate networked observations and all feature the same complement of filters. The majority of these observations were made in SDSS $-i$, with some images taken in Bessell- $V$ and $-R$.

Observations on the $2 \mathrm{~m}$ network telescopes made use of the Spectral imagers, which are also $4096 \times 4096$ pixel Fairchild CCD486 CCDs but have a field of view of $10.5 \mathrm{arcmin}^{2}$, and a pixel scale of $0.304 \operatorname{arcsec}_{\text {pixel }}^{-1}$ in bin $2 \times 2$ mode.

LCOGT operates a network-wide scheduler, which dynamically allocates resources to meet observation requests in real time. The advantage of this system lies in its robust and graceful accommodation of outages due to weather or technical problems at any given telescope. Observations are immediately and automatically re-assigned to an alternative telescope wherever possible.

The RoboNet microlensing programme exploits this flexibility in real-time with a system of software designed to respond automatically to digital alerts of transient phenomena (Tsapras et al. 2009). Based on all available data (from both surveys and followup campaigns), the SIGNALMEN anomaly detector (Dominik et al. 2007), part of the Automated Robotic Terrestrial Exoplanet Microlensing Search (ARTEMiS) system (Dominik et al. 2008a,b), quasi-continuously produces up-to-date point-source-single-lens models of all microlensing events, updates being triggered by any new incoming data, while departures of data from such models are flagged as microlensing 'anomalies'. Using a metric to determine the expected return of observing any specific event (Horne et al. 2009; Dominik et al. 2010), a TArget Prioritisation (TAP) algorithm (Hundertmark et al. 2018) then selects those events that are most valuable, giving special attention to anomalies flagged by SIGNALMEN, while considering the time available and the capabilities of the resources. The Observation Control (OBSCONTROL) software interprets TAP's target recommendations into network observing requests and also handles the returned

${ }^{2}$ https://lco.global stream of imaging data, preparing them for reduction. This stage is also fully robotic, depending on LCOGT's ORAC-based pipeline to remove the instrumental signatures from the images prior to DIA performed by a pipeline based around DANDIA (Bramich 2008). The resulting photometric light curves were immediately made available to the community to facilitate event analysis.

\subsection{The MiNDSTEp campaign}

The MiNDSTEp observations were performed from the Danish $1.54 \mathrm{~m}$ telescope at ESO's La Silla observatory in Chile. The telescope is equipped with a two-colour $512 \times 512$ pixel EMCCD camera (Harpsøe et al. 2012; Skottfelt et al. 2015) with 0.09 arcsec pixel $^{-1}$, corresponding to a $45 \operatorname{arcsec} \times 45 \operatorname{arcsec}$ field of view on the sky. A dichroic beam splitter sends light shortward and longward of $655 \mathrm{~nm}$ to a 'visual' and a 'red' camera, respectively, allowing simultaneous two-colour photometry. A second beam splitter sends the light shortward of $466 \mathrm{~nm}$ into a continuous focusing camera. In order to obtain maximum intensity, and since microlensing is achromatic, there are no filters. In this way, the visual and the red colours are determined by the sensitivity function of the CCD plus the combined throughput of the atmosphere and the telescope. Evans et al. (2016) provide the final sensitivity function, a comparison with the Sloan and Johnson systems, as well as the calibration toward stellar parameters. During the 2014 microlensing observations, the camera was operated at $10 \mathrm{~Hz}$ with a gain setting of $300 \mathrm{e}^{-}$/photon, which typically results in photometric accuracy of the order 1 per cent per 2 min spools. The individual frames in each spool are re-centred during the online reduction (corresponding to a 'tip-and-tilt' hardware compensation for the atmospheric turbulence in adaptive optics), and then sorted into 10 quality classes according to point spread function (PSF). Under good weather conditions, the best PSF groups approach the diffraction limit of the telescope. These are used as templates for the reduction of the full set of exposures, which is performed by use of the DanDIA pipeline (Bramich 2008). While real-time photometric data immediately become publicly available, final data sets are prepared after more careful manual inspection of the process and the tuning of parameters in order to optimise the data quality.

Despite the fact that an observer is present for the operation of the Danish $1.54 \mathrm{~m}$ telescope, the monitoring of the sequence of microlensing events during the night is fully automated, with the observer just pressing a 'start microlensing' button on the telescope control system. The telescope then directly follows the target recommendations provided by the ARTEMiS system (Dominik et al. 2008a,b), according to the adopted MiNDSTEp strategy (Dominik et al. 2010) and incorporating any suspected or detected anomalies identified by the SIGNALMEN detector (Dominik et al. 2007).

\subsection{Monitoring the OGLE-2014-BLG-1186 microlensing event}

On 2014 June 20 UTC, the OGLE survey announced the discovery of event OGLE-2014-BLG-1186, at RA $=17^{\mathrm{h}} .41^{\mathrm{m}} .59 .63$, Dec. $=-34.17$ '.18'.1 (J2000), in tile BLG509 of its low-cadence zone (about one observation every one to two nights). The event brightened relatively slowly given a rather long event time-scale of $t_{\mathrm{E}} \sim 100 \mathrm{~d}$ (predicted at that time) as compared to a median of $t_{\mathrm{E}} \sim 20 \mathrm{~d}$ across all Galactic bulge microlensing events. OGLE2014-BLG-1186 achieved a sufficient priority to make it into the list of events to be monitored by RoboNet and MiNDSTEp consistently both on 2014 September 20 UTC. At that time of the year, the 
Galactic bulge remains low above the horizon from the observing sites, limiting the target visibility to at most $\sim 4 \mathrm{~h}$ per night.

The SIGNALMEN anomaly detector first spotted behaviour not matching the predictions based on real-time RoboNet data on 2014 September 22 UTC, and consequently an e-mail alerting all teams carrying out regular Galactic bulge microlensing observations was circulated. On 2014 September 26 UTC, SIGNALMEN then concluded that a microlensing anomaly was in progress, automatically triggering more intense follow-up from the RoboNet and MiNDSTEp campaigns, as well as fully-automated real-time binary-lens model analysis of the light-curve data by the RTMODEL system, ${ }^{3}$ run at the University of Salerno and based on the VBBINARYLENSING contour integration code (Bozza 2010). Rather than just providing a single best-fitting model, RTMODEL produces a range of alternatives, which narrows down as the anomaly progresses. While initially following the SIGNALMEN trigger, a large variety of models appeared to match the data reasonably well, by 2014 October 6 UTC, it was only models with a mass ratio corresponding to a planet orbiting the lens star that remained feasible (V. Bozza, private communication). An independent assessment (C. Han, private communication) arrived at the same conclusion by 2014 October 20 UTC. The detection timeline of the features of OGLE-2014-BLG1186 is illustrated in Fig. 1 along with the acquired data.

Our preliminary analyses left us with substantial apparent discrepancies between the models and some of the acquired data, and most notably, OGLE and RoboNet data appeared to favour different scenarios. We therefore had to consider the possibility that the putative planetary 'signal' was due to systematic noise in the data. Consequently, this prompted a more careful analysis of the photometric noise in order to be able to consistently claim a signal and to ensure a meaningful interpretation (or to rather reject such a claim).

As it turned out, SIGNALMEN concluded anomalous behaviour being in progress based on the prominent annual parallax signature (due to the Earth's revolution), causing an asymmetry between the rising and falling wing of the light curve, rather than on binarity. Unfortunately, 2014 September 28 UTC was the last night of the annual observing season with the Danish $1.54 \mathrm{~m}$ telescope, so that the MiNDSTEp observations missed the binary signature and provided data only on the rising part of the light curve. By the end of the 2014 observing season, the light curve of event OGLE-2014BLG-1186 was still within the falling wing, about 2 mag above the (I-band) baseline magnitude. While a substantial part of the falling wing was missed due to lack of observability of the target from our sites during the southern summer, a further fading was measured over the full course of the 2015 observing season, and it was only in 2016 that the event reached its baseline magnitude, from which it started to depart already in 2013.

Table 1 provides an overview of the photometric data acquired for microlensing event OGLE-2014-BLG-1186.

\section{MODELLING THE PHOTOMETRIC LIGHT CURVE}

\subsection{Methodology}

Our preliminary assessment obviously showed that OGLE-2014BLG-1186 is strongly affected by annual parallax, and there is a putative further deviation near the peak, potentially caused by a planet orbiting the lens star. However, we also found that the data show some substantial systematic noise. Clearly, we must not take noise for a planetary signal, nor must we let noise corrupt the parallax measurement, which provides valuable information on the properties of the lens star and its planet (should there be one).

Given that previous studies have shown that low-level deviations could be due to red noise instead of real signal (Bachelet et al. 2015), we decided to conduct a similar study on the RoboNet data acquired for OGLE-2014-BLG-1186, which correlates and corrects common brightness patterns of stars in the field of view with various quantities (airmass, CCD position, etc.). Using a PYTHON implementation of Bramich \& Freudling (2012), ${ }^{4}$ we found that any systematics are at least one magnitude smaller than the deviations around the peak.

We also should not confuse features in the putative anomaly over the peak with features due to parallax. Given the long event time-scale, the parallax signal is clearly evident in the wings of the light curve, and measuring it from the wings alone should give pretty much the same result as measuring it from the full data set. The wing region however is not affected by binarity, considered to cause a visible anomaly over the peak. If we were to find a model for the full light curve that successfully describes the peak region, but suggests a significantly different parallax measurement than the wing region does, we would find a clear indication for our interpretation being inconsistent.

We therefore divide the data set into 'peak' and an 'off-peak' subsets, with visual inspection suggesting to define the 'peak' region as the epoch range $6928.8 \leq \mathrm{HJD}-2450000 \leq 6934.0$. Moreover, we adopt an effective noise model, involving a global systematic error and an error bar scaling factor, while a robust fitting procedure prevents parameter estimates being driven by data outliers. We find it fair to assume that the off-peak region is well described by a point-source single-lens model with annual parallax, so that we can construct an effective model for the data residuals with respect to such a model and subsequently apply it to the peak region. With an established model for the noise, we can then assess the significance of a putative anomaly over the peak. Successively determining dominant model parameters, we therefore find full viable models describing event OGLE-2014-BLG-1186 as follows:

(i) Rough estimation of point-source single-lens parameters from off-peak OGLE data.

(ii) Measurement of parallax parameters from off-peak data by means of robust fitting and simultaneous estimation of global systematic error and error bar scaling factor for each data set.

(iii) Application of the estimated global systematic error and error bar scaling factor to the peak data.

(iv) Assessment whether putative peak anomaly is significantly above noise floor and check for consistency between data sets.

If there is evidence for the putative peak anomaly, we consider binary-lens or binary-source interpretations by

(v) grid search for model parameters characterizing a binary lens and establishment of a complete set of all potential viable solutions,

(vi) robust fitting of point-source binary-lens models to all data, (vii) fitting of finite-source binary-lens models to all data,

(viii) fitting of binary-point-source single-lens models to all data, (ix) fitting of binary-finite-source single-lens models to all data. 


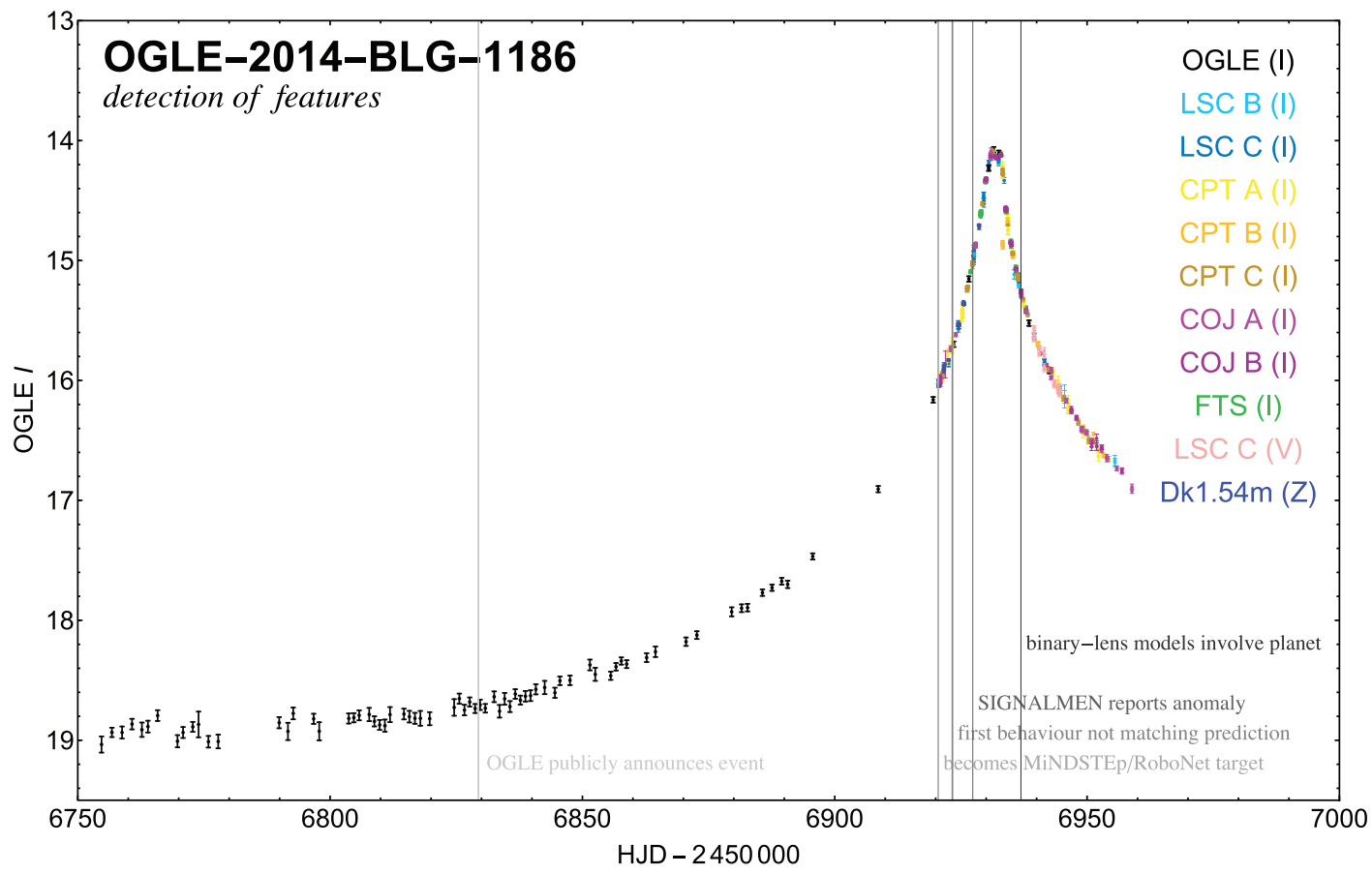

Figure 1. Detection timeline of features of event OGLE-2014-BLG-1186 along with photometric data from various telescopes (colour-coded). The error bars have been adjusted according to the procedure described in Section 3.2 and refer to the $u_{0}<0$ model, while the photometric baseline and blend have been aligned according the $u<0$ close-binary point-source model discussed in Section 3.3. Please note that the SIGNALMEN anomaly detector triggered on the parallax effect apparent in the photometric light curve rather than the binarity.

Table 1. Number of data points acquired with the various telescopes on gravitational microlensing event OGLE-2014-BLG-1186. The 'peak region' is defined as the epoch range $6928.8 \leq \mathrm{HJD}-2450000 \leq 6934.0$.

\begin{tabular}{|c|c|c|c|c|c|c|c|}
\hline \multirow[b]{2}{*}{ Site } & \multirow[b]{2}{*}{ Telescope } & \multirow[b]{2}{*}{ Filter } & \multirow[b]{2}{*}{ Team } & \multirow[b]{2}{*}{ Label } & \multicolumn{3}{|c|}{ Number of data points } \\
\hline & & & & & off-peak & peak & total \\
\hline Las Campanas Observatory & Warsaw $1.3 \mathrm{~m}$ & $I$ & OGLE & OGLE (I) & 642 & 3 & 645 \\
\hline Cerro Tololo Inter-American Observatory (CTIO) & LCO 1m, Dome B & $I$ & RoboNet & LSC B (I) & 40 & 4 & 44 \\
\hline Cerro Tololo Inter-American Observatory (CTIO) & LCO 1m, Dome C & $I$ & RoboNet & LSC C (I) & 32 & 11 & 43 \\
\hline South African Astronomical Observatory (SAAO) & LCO $1 \mathrm{~m}$, Dome A & $I$ & RoboNet & CPT A (I) & 41 & 4 & 45 \\
\hline South African Astronomical Observatory (SAAO) & LCO 1m, Dome B & $I$ & RoboNet & CPT B (I) & 46 & 6 & 52 \\
\hline South African Astronomical Observatory (SAAO) & LCO 1m, Dome C & $I$ & RoboNet & CPT C (I) & 67 & 18 & 85 \\
\hline Siding Spring Observatory (SSO) & LCO 1m, Dome A & $I$ & RoboNet & COJ A (I) & 78 & 45 & 123 \\
\hline Siding Spring Observatory (SSO) & LCO 1m, Dome B & $I$ & RoboNet & COJ B (I) & 54 & 39 & 93 \\
\hline Haleakala Observatory & Faulkes North $2 \mathrm{~m}$ & $I$ & RoboNet & FTS (I) & 35 & 79 & 114 \\
\hline Cerro Tololo Inter-American Observatory (CTIO) & LCO $1 \mathrm{~m}$, Dome C & $V$ & RoboNet & $\mathrm{LSC} C(\mathrm{~V})$ & 24 & 0 & 24 \\
\hline ESO La Silla Observatory & Danish $1.54 \mathrm{~m}$ & $Z$ & MiNDSTEp & Dk1.54m (Z) & 41 & 0 & 41 \\
\hline total & & & & & 1100 & 209 & 1309 \\
\hline
\end{tabular}

\subsection{Parallax measurement and noise model}

\subsubsection{Ordinary microlensing light curves}

A light ray passing a body of mass $M$ at the impact distance $\xi$ experiences a gravitational bending by the angle (Einstein 1915)

$\alpha(\xi)=\frac{4 G M}{c^{2} \xi}$,

where $G$ is the universal gravitational constant, and $c$ is the vacuum speed of light. If we observe a background object ('source') at distance $D_{\mathrm{S}}$ in close angular proximity to the deflecting body ('lens') at distance $D_{\mathrm{L}}$, it appears at angular image positions $x_{i} \theta_{\mathrm{E}}$, measured relative to the lens position, rather than its true angular position $u \theta_{\mathrm{E}}$, related by

$u(x)=x-\frac{1}{x}$,

with $\theta_{E}$ being the angular Einstein radius

$\theta_{\mathrm{E}}=\sqrt{\frac{4 G M}{c^{2}} \frac{\pi_{\mathrm{LS}}}{1 \mathrm{au}}}$,

where

$\pi_{\mathrm{LS}}=1$ au $\left(D_{\mathrm{L}}^{-1}-D_{\mathrm{S}}^{-1}\right)$

is the relative parallax of lens and source with respect to the observer.

Gravitational microlensing events show a transient brightening of an observed source star that results from the gravitational bending of its light by an intervening object, which follows from equation (3) 
as

$A(u)=\sum_{i}\left|\frac{u\left(x_{i}\right)}{x_{i}} \frac{\mathrm{d} u}{\mathrm{~d} x}\left(x_{i}\right)\right|^{-1}$

For single point-like source and lens stars, one finds two images

$x_{1 / 2}=\frac{1}{2}\left(u \pm \sqrt{u^{2}+4}\right)$,

so that the observed magnification, equation (6), evaluates to the analytic expression (Einstein 1936)

$A(u)=\frac{u^{2}+2}{u \sqrt{u^{2}+4}}$.

If we assume a uniform relative proper motion $\mu$ between lens and source star, the separation parameter $u$ becomes (Paczyński 1986)

$u\left(t ; t_{0}, u_{0}, t_{\mathrm{E}}\right)=\sqrt{u_{0}^{2}+\left(\frac{t-t_{0}}{t_{\mathrm{E}}}\right)^{2}}$,

where $t_{\mathrm{E}}=\theta_{\mathrm{E}} / \mu$ is the event time-scale, and the closest angular approach $u_{0} \theta_{\mathrm{E}}$ is realised at time $t_{0}$.

With $F_{\mathrm{S}}^{[j]}$ being the unmagnified flux of the observed target star, and $F_{\mathrm{B}}^{[j]}$ the flux contributed by other light sources, corresponding to a specific detector and labelled by the index $m$, the total observed flux becomes

$$
\begin{aligned}
F^{[j]}(t) & =F_{\mathrm{S}}^{[j]} A[u(t ; \boldsymbol{p})]+F_{\mathrm{B}}^{[j]} \\
& =F_{\mathrm{S}}^{[j]}\{A[u(t ; \boldsymbol{p})]-1\}+F_{\text {base }}^{[j]},
\end{aligned}
$$

where $F_{\text {base }}^{[j]}=F_{\mathrm{S}}^{[j]}+F_{\mathrm{B}}^{[j]}$ is the baseline flux and $\boldsymbol{p}$ denotes the set of parameters characterising the magnification function $A[u(t ; \boldsymbol{p})]$. The total flux can also be written as

$F^{[j]}(t)=F_{\text {base }}^{[j]} A_{\text {obs }}^{[j]}(t ; \boldsymbol{p})$,

where

$A_{\mathrm{obs}}^{[j]}[u(t ; \boldsymbol{p})]=\frac{A[u(t ; \boldsymbol{p})]+g^{[j]}}{1+g^{[j]}}$

is the observed magnification, with

$g^{[j]}=F_{\mathrm{B}}^{[j]} / F_{\mathrm{S}}^{[j]}=F_{\text {base }}^{[j]} / F_{\mathrm{S}}^{[j]}-1$

being the blend ratio for the given detector.

Because of $A(u)$ monotonically increasing as $u \rightarrow 0$, the light curves of ordinary microlensing events, assuming a single isolated lens star and a point-like source star as well as uniform relative proper motion, reach a peak at $t_{0}$, where the closest angular approach between lens and source $u\left(t_{0}\right)=u_{0}$ is realised, and are symmetric in time with respect to this peak. They are fully characterised by $\boldsymbol{p}=\left(t_{0}, u_{0}, t_{\mathrm{E}}\right)$ and the set of $\left(F_{\text {base }}^{[j]}, g^{[j]}\right)$ for each detector. While $\left(F_{\text {base }}^{[j]}, F_{\mathrm{S}}^{[j]}\right)$ follow analytically from linear regression, the magnification function $A[u(t ; \boldsymbol{p})]$ is generally non-linear in the parameters $\boldsymbol{p}$.

\subsubsection{Annual parallax}

An annual parallax effect is caused by the revolution of the Earth, leading to a change of the line of sight, which alters the observed microlensing magnification. Let $\gamma(t)$ (1 au) denote the projection of the Earth's orbit on to a plane perpendicular to the direction towards the source star. With $\boldsymbol{\mu}_{\mathrm{S}}$ and $\boldsymbol{\mu}_{\mathrm{L}}$ denoting the proper motions of the source and lens stars, respectively, while $\pi_{\mathrm{S}}$ and $\pi_{\mathrm{L}}$ denote their parallaxes, the apparent geocentric positions of source and lens star may be written (c.f. An et al. 2002; Gould 2004) as

$\theta_{\mathrm{S}}(t)=\theta_{\mathrm{S}, 0}+\left(t-t_{0}\right) \mu_{\mathrm{S}}-\pi_{\mathrm{S}} \gamma(t)$,

$\theta_{\mathrm{L}}(t)=\theta_{\mathrm{L}, 0}+\left(t-t_{0}\right) \mu_{\mathrm{L}}-\pi_{\mathrm{L}} \gamma(t)$,

so that

$\boldsymbol{\theta}(t) \equiv \boldsymbol{\theta}_{\mathrm{S}}(t)-\boldsymbol{\theta}_{\mathrm{L}}(t)=\left(\boldsymbol{\theta}_{\mathrm{S}}-\boldsymbol{\theta}_{\mathrm{L}}\right)_{0}-\left(t-t_{0}\right) \boldsymbol{\mu}_{\mathrm{LS}}+\pi_{\mathrm{LS}} \boldsymbol{\gamma}(t)$,

with $\boldsymbol{\mu}_{\mathrm{LS}} \equiv \boldsymbol{\mu}_{\mathrm{L}}-\boldsymbol{\mu}_{\mathrm{S}}$ and $\pi_{\mathrm{LS}} \equiv \pi_{\mathrm{L}}-\pi_{\mathrm{S}}$ denoting the relative proper motion and relative parallax between lens and source, while $\left(\boldsymbol{\theta}_{\mathrm{S}}-\boldsymbol{\theta}_{\mathrm{L}}\right)_{0} \equiv \boldsymbol{\theta}_{\mathrm{S}, 0}-\boldsymbol{\theta}_{\mathrm{L}, 0}$.

Hence, for $\boldsymbol{u}(t)=\boldsymbol{\theta}(t) / \theta_{\mathrm{E}}$ we find with the microlensing parallax parameter $\pi_{\mathrm{E}} \equiv \pi_{\mathrm{LS}} / \theta_{\mathrm{E}}$,

$\boldsymbol{u}(t)=\boldsymbol{u}_{0}+\left(t-t_{0}\right) \dot{\boldsymbol{u}}_{0}+\pi_{\mathrm{E}} \delta \boldsymbol{\gamma}(t)$,

where

$\boldsymbol{u}_{0} \equiv \boldsymbol{u}\left(t_{0}\right)=\frac{\left(\boldsymbol{\theta}_{\mathrm{S}}-\boldsymbol{\theta}_{\mathrm{L}}\right)_{0}}{\theta_{\mathrm{E}}}+\pi_{\mathrm{E}} \boldsymbol{\gamma}\left(t_{0}\right)$,

$\dot{\boldsymbol{u}}_{0} \equiv \dot{\boldsymbol{u}}\left(t_{0}\right)=-\frac{\boldsymbol{\mu}_{\mathrm{LS}}}{\theta_{\mathrm{E}}}+\pi_{\mathrm{E}} \dot{\boldsymbol{\gamma}}\left(t_{0}\right)$,

$\delta \boldsymbol{\gamma}(t)=\boldsymbol{\gamma}(t)-\boldsymbol{\gamma}\left(t_{0}\right)-\left(t-t_{0}\right) \dot{\gamma}\left(t_{0}\right)$.

Given that by construction $\delta \boldsymbol{\gamma}\left(t_{0}\right)=0$ and $\delta \dot{\gamma}\left(t_{0}\right)=0$, one explicitly sees that for epochs near $t_{0}$, the lowest order local effect of the annual parallax distorting the symmetric light curve of a single lens arises from the Earth's acceleration along its orbit, corresponding to the curvature of the effective source trajectory $\boldsymbol{u}(t) \theta_{\mathrm{E}}$.

With $\left(\hat{\boldsymbol{e}}_{\mathrm{n}}, \hat{\boldsymbol{e}}_{\mathrm{e}}\right)$ denoting unit vectors in the direction of ecliptic north and east, respectively,

$\delta \boldsymbol{\gamma}(t)=\delta \gamma_{\mathrm{n}}(t) \hat{\boldsymbol{e}}_{\mathrm{n}}+\delta \gamma_{\mathrm{e}}(t) \hat{\boldsymbol{e}}_{\mathrm{e}}$,

while $\boldsymbol{u}(t)$ can be written in terms of components parallel and perpendicular to the effective source trajectory as

$$
\begin{gathered}
u_{\|}\left(t ; t_{0}, t_{\mathrm{E}}, \pi_{\mathrm{E}}\right)=\frac{t-t_{0}}{t_{\mathrm{E}}}+\pi_{\mathrm{E}, \mathrm{N}} \delta \gamma_{\mathrm{n}}(t)+\pi_{\mathrm{E}, \mathrm{E}} \delta \gamma_{\mathrm{e}}(t), \\
u_{\perp}\left(t ; u_{0}, \pi_{\mathrm{E}}\right)=u_{0}-\pi_{\mathrm{E}, \mathrm{E}} \delta \gamma_{\mathrm{n}}(t)+\pi_{\mathrm{E}, \mathrm{N}} \delta \gamma_{\mathrm{e}}(t),
\end{gathered}
$$

where $t_{\mathrm{E}}=\theta_{\mathrm{E}} /|\boldsymbol{\mu}|$ with

$\boldsymbol{\mu}=\dot{\boldsymbol{u}}_{0} \theta_{\mathrm{E}}=-\boldsymbol{\mu}_{\mathrm{LS}}+\pi_{\mathrm{E}} \theta_{\mathrm{E}} \dot{\boldsymbol{\gamma}}\left(t_{0}\right)$

and

$\pi_{\mathrm{E}}=\sqrt{\pi_{\mathrm{E}, \mathrm{N}}^{2}+\pi_{\mathrm{E}, \mathrm{E}}^{2}}$

so that $\left(\pi_{E, N}, \pi_{E, E}\right)$ form components of a vector $\pi_{E}$.

Hence, accounting for annual parallax, the microlensing light curve due to a single lens star can be characterised by the parameters $\boldsymbol{p}=\left(t_{0}, u_{0}, t_{\mathrm{E}}, \pi_{\mathrm{E}, \mathrm{N}}, \pi_{\mathrm{E}, \mathrm{E}}\right)$, with the magnification given by equation (8) and

$u\left(t ; t_{0}, u_{0}, t_{\mathrm{E}}, \boldsymbol{\pi}_{\mathrm{E}}\right)=\sqrt{\left[u_{\|}\left(t ; t_{0}, t_{\mathrm{E}}, \boldsymbol{\pi}_{\mathrm{E}}\right)\right]^{2}+\left[u_{\perp}\left(t ; u_{0}, \boldsymbol{\pi}_{\mathrm{E}}\right)\right]^{2}}$.

\subsubsection{Noise model for photometric measurements and robust fitting}

Let us consider $M$ data sets, one for each detector, labelled by the index $j \in\{1, \ldots, M\}$, containing $N^{[j]}$ data points, respectively, 
labelled by the index $i \in\left\{1, \ldots, N^{[j]}\right\}$, so that the data tuple $\left(t_{i}^{[j]}, F_{i}^{[j]}, \sigma_{i}^{[j]}\right)$ denotes the time the measurement was taken, the measured flux, and the uncertainty of the measured flux.

In order to describe the measurement uncertainties of our photometric data, we adopt a model that combines error bar rescaling with a robust-fitting procedure that applies weights to effectively correct for outliers and wide tails.

Similar to Tsapras et al. (2003), we adopt a scaling factor $\kappa^{[j]}$ for the reported uncertainty $\sigma_{i}^{[j]}$, as well as a constant fractional systematic uncertainty $s_{0}^{[j]}$ in the reported flux $F_{i}^{[j]}$ (equivalent to a constant systematic uncertainty in the reported magnitude), so that

$\tilde{\sigma}_{i}^{[j]}\left(\sigma_{i}^{[j]}, \kappa^{[j]}, s_{0}^{[j]}\right)=\sqrt{\left(\kappa^{[j]} \sigma_{i}^{[j]}\right)^{2}+\left(s_{0}^{[j]} F_{i}^{[j]}\right)^{2}}$

is assumed to represent the standard deviation of a Gaussian distribution. This leads to the standardised residuals

$r_{i}^{[j]}\left(F^{[j]}\left(t_{i}^{[j]}\right), F_{i}^{[j]}, \tilde{\sigma}_{i}^{[j]}\right)=\frac{F_{i}^{[j]}-F^{[j]}\left(t_{i}^{[j]}\right)}{\tilde{\sigma}_{i}^{[j]}}$.

With the modified uncertainties $\tilde{\sigma}_{i}^{[j]}$ depending on the parameters $\kappa^{[j]}$ and $s_{0}^{[j]}$, a maximum-likelihood estimate is then obtained by minimising

$\tilde{\chi}^{2}=\sum_{i=1}^{M} \sum_{j=1}^{N^{[j]}}\left[\left(r_{i}^{[j]}\right)^{2}+2 \ln \tilde{\sigma}_{i}^{[j]}\right]$,

which is a modification of the ordinary $\chi^{2}$, which differs by an additional term due to the non-constant $\tilde{\sigma}_{i}^{[j]}$ and does not follow $\chi^{2}$ statistics.

Accounting for scaling factors $\kappa^{[j]}$ and systematic uncertainties $s_{0}^{[j]}$ according to equation (25) does not account for the distribution of the standardised residuals being more tail-heavy than a Gaussian distribution. While this could be achieved by using Student's $t$ distribution (with an additional parameter), we adopt a procedure that uses a pseudo-Gaussian distribution involving a weight factor, similar to that used by the SIGNALMEN anomaly detector (Dominik et al. 2007). Robust fitting procedures (e.g. Hoaglin, Mosteller \& Tukey 1983; Huber \& Ronchetti 2009) enforce the model function $F^{[j]}(t)$ to follow the bulk of the data rather than being substantially effected by outliers in the data set. Like Dominik et al. (2007), we determine the median of the absolute standardised residuals $\tilde{r}^{[j]}$ and apply a bi-square weight

$w_{i}^{[j]}=\left\{\begin{array}{cc}{\left[1-\left(\frac{r_{i}^{[j]}}{K \tilde{r}^{[j]}}\right)^{2}\right]^{2}} & \text { for }\left|r_{i}^{[j]}\right|<K \tilde{r}^{[j]} \\ 0 & \text { for }\left|r_{i}^{[j]}\right| \geq K \tilde{r}^{[j]}\end{array}\right.$

to each data point, where we adopt $K=6$ for the tuning constant. In principle, we could have chosen $\beta \equiv K^{-1}$ as a further free parameter, with $\beta=0$ corresponding to a Gaussian without any data downweighting, i.e. $w_{i}^{[j]}=1$ for all $n$. However, $\beta$ is not strictly constrained by our data, and thus the exact choice does not make a significant difference, and we can accept that our procedure would enforce downweighting even to data that perfectly match a Gaussian distribution. We explicitly choose a continuous weight function in order to ensure that our numerical minimization procedures behave well rather than getting confused by discontinuities. The weight $w_{i}^{[j]}$ becomes zero for data points whose absolute standardised residuals exceeds $K$ times their median.
With the weights $w_{i}^{[j]}$, we estimate model parameters by minimising

$\tilde{\chi}^{2}=\sum_{i=1}^{M} \sum_{j=1}^{N^{[j]}} w_{i}^{[j]}\left[\left(r_{i}^{[j]}\right)^{2}+2 \ln \tilde{\sigma}_{i}^{[j]}\right]$,

which is repeated for subsequent sets of standardised residuals until $\tilde{\chi}^{2}$ converges.

\subsubsection{Off-peak parallax model for OGLE-2014-BLG-1186}

We used the modelling capabilities of the SIGNALMEN anomaly detector (Dominik et al. 2007), which itself calls the CERN library routine MINUIT (James \& Roos 1975) for non-linear minimisation, in order to fit a point-source single-lens parallax model to the offpeak data while establishing an effective noise model of our data.

A rough estimate of the fundamental parameters $\left(t_{0}, u_{0}, t_{E}\right)$ can be obtained from simple maximum-likelihood fitting of a point-source single-lens model to the OGLE data, starting at any seed that roughly locates the peak, e.g. $\left(t_{0}, u_{0}, t_{\mathrm{E}}\right)=(6932.0,0.3,20 \mathrm{~d})$. This gave us the parameters listed in the first column of Tables 3 and 4, which were then used to construct seeds for models including the annual parallax, where, in order to account for potential ambiguities, we used all permutations of signs for the parameters $\left(u_{0}, \pi_{\mathrm{E}, \mathrm{N}}, \pi_{\mathrm{E}, \mathrm{E}}\right)$, specifically $\left(u_{0}, \pi_{\mathrm{E}, \mathrm{N}}, \pi_{\mathrm{E}, \mathrm{E}}\right)=( \pm 0.009275, \pm 0.1, \pm 0.1)$. Using the robust fitting procedure with the noise model outlined above, i.e. by minimising $\tilde{\chi}^{2}$ as defined by equation (29), we found two classes of local minima, corresponding to a 'good' fit with $\chi^{2} \sim$ 1050 for 645 data points with $t_{\mathrm{E}} \sim 300 \mathrm{~d}$ and a 'bad' fit with $\chi^{2}$ $\sim 3050$ for 645 data points with $t_{\mathrm{E}} \sim 180 \mathrm{~d}$. We accepted the former and rejected the latter due to not reasonably matching the data. This left us with the two viable options $\left(u_{0}, \pi_{\mathrm{E}, \mathrm{N}}, \pi_{\mathrm{E}, \mathrm{E}}\right)=(-$ $0.0052 \pm 0.0018,-0.367 \pm 0.012,-0.143 \pm 0.015)$ and $\left(u_{0}, \pi_{\mathrm{E}, \mathrm{N}}\right.$, $\left.\pi_{\mathrm{E}, \mathrm{E}}\right)=(0.0054 \pm 0.0017,-0.354 \pm 0.010,-0.138 \pm 0.014)$, distinguished by the sign of $u_{0}$.

While the OGLE data provides a coverage of all event phases (except for the epochs that correspond to the gaps in between the annual seasons) and therefore should provide a good estimate of the parallax parameters, other data sets cover the event more densely over substantial parts of the wings, but all data might suffer from some systematics. With all data sets, except for the Danish $1.54 \mathrm{~m}$ (which cover only the rising part and therefore lack of relevant information), we find $\left(u_{0}, \pi_{\mathrm{E}, \mathrm{N}}, \pi_{\mathrm{E}, \mathrm{E}}\right)=$ $(-0.0065 \pm 0.0004,-0.354 \pm 0.009,-0.178 \pm 0.008)$ and $\left(u_{0}\right.$, $\left.\pi_{\mathrm{E}, \mathrm{N}}, \pi_{\mathrm{E}, \mathrm{E}}\right)=(0.0061 \pm 0.0004,-0.343 \pm 0.009,-0.165 \pm 0.009)$, so that the parallax appears to be robustly measured, with the further data giving a tighter constraint. We determined the error bar rescaling for the Danish $1.54 \mathrm{~m}$ data based on these models.

In Table 2, we report the inferred systematic errors $s_{0}^{[j]}$ and scaling factors $\kappa^{[j]}$ for the various data sets, based on the standardised residuals of the two robust single-lens point-source models with parallax to all data (except for the Danish $1.54 \mathrm{~m}$ ), while Fig. 2 shows the weighted cumulative distribution functions (CDFs) of the standardised residuals and $\mathrm{CDF}$ of the data weights, quoting $p$-values of an Anderson-Darling (AD) test (Anderson \& Darling 1952) comparing the weighted distribution of standardised residuals with a standard Gaussian. Some of the reported uncertainties on $s_{0}^{[j]}$ and $\kappa^{[j]}$ are large, and for some of the data sets, we find an ambiguity between the systematic error and the scaling factor. In fact, if the reported error bars on the magnitude do not vary much, there is no difference between adding a systematic error in quadrature and 
Table 2. Adopted error bar scaling factor $\kappa$ and systematic error $s_{0}$ for the various data sets, as defined by equation (25), determined from the standardised residuals arising for the point-source single-lens parallax models to all off-peak data (except for Danish $1.54 \mathrm{~m}$ ) for $u_{0}<0$ or $u_{0}>0$, respectively, whose parameters are listed in Tables 3 and 4 . Range constraints $\kappa \geq 0.1$ and $s_{0} \geq 10^{-5}$ have been adopted, and the asterisk $\left(^{\star}\right)$ marks bouncing against the range boundary. Several data sets do not hold sufficient information to constrain both $\kappa$ and $s_{0}$, leaving us with parameter ambiguities for our effective noise model.

\begin{tabular}{|c|c|c|c|c|c|}
\hline & & \multicolumn{2}{|c|}{$u_{0}<0$} & \multicolumn{2}{|c|}{$u_{0}>0$} \\
\hline & & $\kappa$ & $s_{0}$ & $\kappa$ & $s_{0}$ \\
\hline OGLE & I & $0.99 \pm 0.07$ & $0.021 \pm 0.006$ & $0.99 \pm 0.07$ & $0.021 \pm 0.005$ \\
\hline LSC B & I & $3.8 \pm 0.5$ & $10^{-5}\left(^{\star}\right)$ & $3.8 \pm 0.5$ & $10^{-5}\left(^{\star}\right)$ \\
\hline LSC C & I & $0.1\left(^{\star}\right)$ & $0.022 \pm 0.003$ & $0.1\left(^{\star}\right)$ & $0.023 \pm 0.003$ \\
\hline CPT A & I & $0.1\left(^{\star}\right)$ & $0.032 \pm 0.004$ & $0.1\left(^{\star}\right)$ & $0.032 \pm 0.004$ \\
\hline СРТ B & I & $0.1\left(^{\star}\right)$ & $0.0124 \pm 0.0014$ & $0.1\left(^{\star}\right)$ & $0.0123 \pm 0.0014$ \\
\hline СPT C & I & $1.10 \pm 0.16$ & $0.004 \pm 0.003$ & $1.08 \pm 0.16$ & $0.004 \pm 0.003$ \\
\hline $\mathrm{COJ} \mathrm{A}$ & I & $1.5 \pm 0.2$ & $0.002 \pm 0.003$ & $1.5 \pm 0.2$ & $0.002 \pm 0.004$ \\
\hline $\mathrm{COJ} \mathrm{B}$ & I & $1.51 \pm 0.16$ & $10^{-5}\left(^{\star}\right)$ & $1.45 \pm 0.15$ & $10^{-5}\left(^{\star}\right)$ \\
\hline FTS & I & $0.1\left(^{\star}\right)$ & $0.0094 \pm 0.0012$ & $0.1\left(^{\star}\right)$ & $0.0094 \pm 0.0012$ \\
\hline LSC C & V & $0.30 \pm 0.05$ & $10^{-5}\left({ }^{\star}\right)$ & $0.30 \pm 0.05$ & $10^{-5}\left({ }^{\star}\right)$ \\
\hline Dk1.54m & Z & $0.8 \pm 0.3$ & $0.003 \pm 0.002$ & $0.8 \pm 0.3$ & $0.004 \pm 0.002$ \\
\hline
\end{tabular}

Table 3. Successive construction of models for $u_{0}<0$ in five steps: (1) rough maximum-likelihood estimation of $t_{0}$, $t_{\mathrm{E}}$, and $u_{0}$ from the off-peak OGLE data on the basis of the reported error bars and a single-lens point source model, (2) measurement of parallax parameters from the off-peak OGLE data (assuming $u_{0}<0$ ) by means of robust fitting and simultaneous estimation of global systematic error and error bar scaling factor, with refinement of $t_{0}, t_{\mathrm{E}}$, and $u_{0}$ estimates, (3) confirmation of robustness of parallax measurement and refinement of parameters by including all off-peak data (except for Danish $1.54 \mathrm{~m}$ ), followed by determination of the systematic error and error bar scaling factor for the Danish $1.54 \mathrm{~m}$ data based on the arising model parameters, (4) inclusion of the peak data using the established modification of error bars, and robust fitting of a binary-lens point-source model to all data (including Danish $1.54 \mathrm{~m})$, with seed values for the binary parameters $(d, q, \alpha)$ arising from a grid search with the other parameters fixed, (5) finding a corresponding solution with a wide binary lens $(d>1$ rather than $d<1)$ by using the previously determined parameter values as seed, and just flipping the separation parameter $d \leftrightarrow d^{-1}$.

\begin{tabular}{|c|c|c|c|c|c|}
\hline Model & Single & Single, parallax & Single, parallax & Binary, parallax & Binary, parallax \\
\hline Data selection & Off-peak & Off-peak & Off-peak & All & All \\
\hline Data sets & OGLE (I) & OGLE (I) & All except Dk1.54m & All & All \\
\hline Data scaling & None & None & None & $u_{0}<0$ off-peak & $u_{0}<0$ off-peak \\
\hline Option & - & $u_{0}<0$ & $u_{0}<0$ & $u_{0}<0$, close & $u_{0}<0$, wide \\
\hline$t_{0}$ & $6931.685 \pm 0.005$ & $6931.39 \pm 0.09$ & $6931.359 \pm 0.006$ & $6931.429 \pm 0.003$ & $6931.477 \pm 0.003$ \\
\hline$\pi_{\mathrm{E}, \mathrm{N}}$ & - & $-0.367 \pm 0.012$ & $-0.354 \pm 0.009$ & $-0.364 \pm 0.009$ & $-0.353 \pm 0.007$ \\
\hline$\pi_{\mathrm{E}, \mathrm{E}}$ & - & $-0.143 \pm 0.015$ & $-0.178 \pm 0.008$ & $-0.171 \pm 0.009$ & $-0.171 \pm 0.006$ \\
\hline$d$ & - & - & - & $0.713 \pm 0.006$ & $1.428 \pm 0.009$ \\
\hline$q$ & - & - & - & $(3.6 \pm 0.3) \times 10^{-4}$ & $(3.8 \pm 0.2) \times 10^{-4}$ \\
\hline$\alpha$ & - & - & - & $4.023 \pm 0.002$ & $4.022 \pm 0.002$ \\
\hline
\end{tabular}

scaling the error bars by a common factor. For some data sets, the photometric uncertainty can pretty much be described just by a constant systematic error, regardless of the reported error bar, while for some other data sets, a systematic error is rejected, but a substantial scaling factor is suggested. For most data sets, the small number of data points prevents the establishment of a noise model that is more detailed than a simple effective model, particularly given the small number of large absolute standardised residuals (which are relevant in order to provide such statistics). Comparing the CDF of the weighted standardised residuals with a Gaussian distribution (see Fig. 2) shows that our effective model provides a reasonable description. The distribution of the weights reveals that the distribution of the standardised residuals is generally more tail-heavy than a Gaussian distribution, where the weight of the tail differs amongst the data sets. Hence, a Gaussian profile with just an increased error bar would not be a good description. However, a student- $t$ distribution would provide an alternative to our adopted weight function.

It is worth stressing that we adopt a simple effective model for describing the measurement uncertainties such that these reasonable match the acquired data. We neither claim that our specific choice is without alternatives nor that it is the most appropriate one. We find that the parameters of our model are already rather poorly constrained, while we in particular neglect any dependence of the statistics on the brightness of the object. By essentially adding a constant systematic uncertainty to the magnitude, we may overestimate the uncertainty as our target brightens, but our main goal is in not underestimating the uncertainty so that 'noise' patterns are not mistaken as signals. The reported $s_{0}=0.021 \pm 0.006$ for OGLE seems rather large, but its uncertainty is substantial and it is not dramatically out of line with other observatories. Its estimation is dominated by the measurements of the unbrightened 
Table 4. Successive construction of models for $u_{0}>0$, analogous to the $u_{0}<0$ case presented in Table 3 . Step 1 is identical to the procedure for $u_{0}<0$ (given that it the single-lens point-source light curve without parallax depends on $\left|u_{0}\right|$ only), whereas for the other steps the opposite sign for $u_{0}$ has been enforced, leading to a flip in sign of the trajectory angle $\alpha$ (or respectively $\alpha \leftrightarrow \alpha \pm \pi$ ), while all other parameters differ slightly.

\begin{tabular}{|c|c|c|c|c|c|}
\hline Model & Single & Single, parallax & Single, parallax & Binary, parallax & Binary, parallax \\
\hline Data selection & Off-peak & Off-peak & Off-peak & All & All \\
\hline Data sets & OGLE (I) & OGLE (I) & All except Dk1.54m & All & All \\
\hline Data scaling & None & None & None & $u_{0}>0$ off-peak & $u_{0}>0$ off-peak \\
\hline Option & - & $u_{0}>0$ & $u_{0}>0$ & $u_{0}>0$, close & $u_{0}>0$, wide \\
\hline$t_{0}$ & $6931.685 \pm 0.005$ & $6931.37 \pm 0.09$ & $6931.356 \pm 0.006$ & $6931.444 \pm 0.004$ & $6931.516 \pm 0.005$ \\
\hline$\pi_{\mathrm{E}, \mathrm{N}}$ & - & $-0.354 \pm 0.010$ & $-0.343 \pm 0.009$ & $-0.354 \pm 0.009$ & $-0.352 \pm 0.009$ \\
\hline$\pi_{\mathrm{E}, \mathrm{E}}$ & - & $-0.138 \pm 0.014$ & $-0.165 \pm 0.009$ & $-0.160 \pm 0.009$ & $-0.157 \pm 0.008$ \\
\hline$d$ & - & - & - & $0.681 \pm 0.006$ & $1.483 \pm 0.013$ \\
\hline$q$ & - & - & - & $(4.3 \pm 0.3) \times 10^{-4}$ & $(4.3 \pm 0.3) \times 10^{-4}$ \\
\hline$\alpha$ & - & - & - & $2.308 \pm 0.003$ & $2.305 \pm 0.002$ \\
\hline
\end{tabular}

source, and such a value is not an atypical scatter for OGLE measurements of stars as faint as $I \sim 19$. Therefore, we particularly do not consider it to be indicative of intrinsic variability of the source. A detailed discussion of the photometric uncertainties of the OGLE-IV data has recently been carried out by Skowron et al. (2016).

The respective model light curves for the two single-lens pointsource models with parallax to all data along with the data with modified error bars are shown in Fig. 3 for $u_{0}<0$ and Fig. 4 for $u_{0}>0$, respectively, whereas Tables 3 and 4 list the corresponding model parameters.

\subsubsection{Significance of putative anomaly}

Given our robust measurement of parallax and our noise model from the off-peak data, we can assess the putative anomaly in the peak region, assuming that the inferred systematic errors and scale factors reasonably apply to the peak data as well. If we consider only OGLE data, there is no obvious hint of an anomaly, as illustrated in Fig. 5, which shows single-lens point-source models with parallax for all OGLE data for the two cases $u_{0}<0$ and $u_{0}>0$, respectively.

The situation however becomes dramatically different once one considers the RoboNet data. The top panels of Fig. 6 show the respective single-lens point-source model with parallax for the offpeak data only, along with the peak data, for which the baseline magnitude $F_{\text {base }}^{[j]}$ and blend ratio $g^{[j]}$ also follow the fit to the offpeak data only. Apparently, the RoboNet data over the peak from three telescopes in South Africa and two telescopes in Australia, for which the baseline magnitude and blend ratio are well determined (in contrast to the FTS and Chilean data), consistently line up to very high precision without the modelling process ever having involved these data. Moreover, a microlensing anomaly is clearly visible, much above the noise level.

\subsection{Binary-lens models}

\subsubsection{Constraining binary-lens parameter space}

With the presence of a real anomaly over the peak firmly established, let us systematically find all potentially viable binary-lens models, which include the case of a star orbited by a planet (with the effect of other planets neglected).
Given that the peak anomaly lasts only about $5 \mathrm{~d}$, we can at first neglect the binary orbital motion, assuming that the orbital period is much longer. With regard to its effect on the gravitational bending of light, a binary lens composed of constituents with masses $M_{1}$ and $M_{2}$ is then fully characterised by its total mass $M=M_{1}+M_{2}$, the mass ratio $q=M_{2} / M_{1}$, and the separation parameter $d$, where $d \theta_{\mathrm{E}}$ is the angle on the sky between the primary and the secondary as seen from the observer with the angular Einstein radius $\theta_{\mathrm{E}}$, as given by equation (4), referring to the total mass $M$.

Let us choose a coordinate frame with the origin at the centre of mass of the lens system and the coordinate axes $\left(\boldsymbol{e}_{1}, \boldsymbol{e}_{2}\right)$ spanning a plane orthogonal to the line of sight so that $\boldsymbol{e}_{1} \perp \boldsymbol{e}_{2}$ and $\boldsymbol{e}_{1} \times \boldsymbol{e}_{2}$ points towards the observer. With $\boldsymbol{e}_{1}$ being along the orthogonally projected separation vector from $M_{2}$ to $M_{1}$, the primary of mass $M_{1}$ is at the angular coordinate $[d q /(1+q), 0] \theta_{\mathrm{E}}$ and the secondary of mass $M_{2}$ is at the angular coordinate $[-d /(1+q), 0] \theta_{\mathrm{E}}$.

In contrast to a single lens, the microlensing light curve depends on the orientation of the source trajectory, where we measure the trajectory angle $\alpha$ from the axis $\boldsymbol{e}_{1}$. We can then describe the source trajectory by

$\boldsymbol{u}(t)=u_{0}\left(\begin{array}{c}-\sin \alpha \\ \cos \alpha\end{array}\right)+\frac{t-t_{0}}{t_{\mathrm{E}}}\left(\begin{array}{c}\cos \alpha \\ \sin \alpha\end{array}\right)$,

where the source most closely approaches the centre of mass of the lens system at epoch $t_{0}$ and angular separation $u_{0} \theta_{\mathrm{E}}$.

For weak gravitational fields, one finds a linear superposition of the deflection terms that arise for each point-like deflector with mass $M_{k}$ at angular position $\boldsymbol{x}^{(k)} \theta_{\mathrm{E}}$, so that the relation between the source and image positions (cf. equation 3 ) becomes

$\boldsymbol{u}(\boldsymbol{x})=\boldsymbol{x}-\sum_{k} \frac{M_{k}}{M} \frac{\boldsymbol{x}-\boldsymbol{x}^{(k)}}{\left|\boldsymbol{x}-\boldsymbol{x}^{(k)}\right|^{2}}$,

while the magnification is given by

$A(\boldsymbol{u})=\sum_{i}\left|\operatorname{det}\left(\frac{\partial \boldsymbol{u}}{\partial \boldsymbol{x}}\right)\left(\boldsymbol{x}_{i}\right)\right|^{-1}$,

where the sum is taken over all images at angular positions $\boldsymbol{x}_{\boldsymbol{i}} \theta_{\mathrm{E}}$. Binary (and multiple) lenses create line caustics $\mathcal{C}$, defined by

$\mathcal{C}=\left\{\boldsymbol{u}\left(\boldsymbol{x}^{\prime}\right) \mid \operatorname{det}\left(\frac{\partial \boldsymbol{u}}{\partial \boldsymbol{x}}\right)\left(\boldsymbol{x}^{\prime}\right)=0\right\}$

on which the point-source magnification diverges, $A(\boldsymbol{u}) \rightarrow \infty$. The features of the diverse morphologies of microlensing light curves 


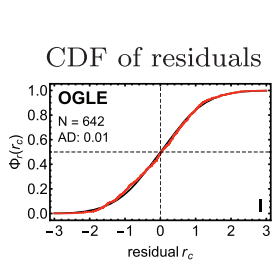

$u_{0}<0$
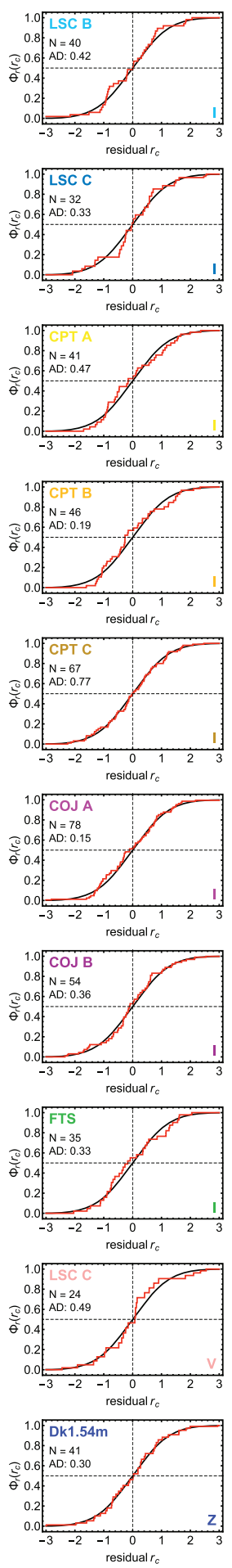

$\mathrm{CDF}$ of weights
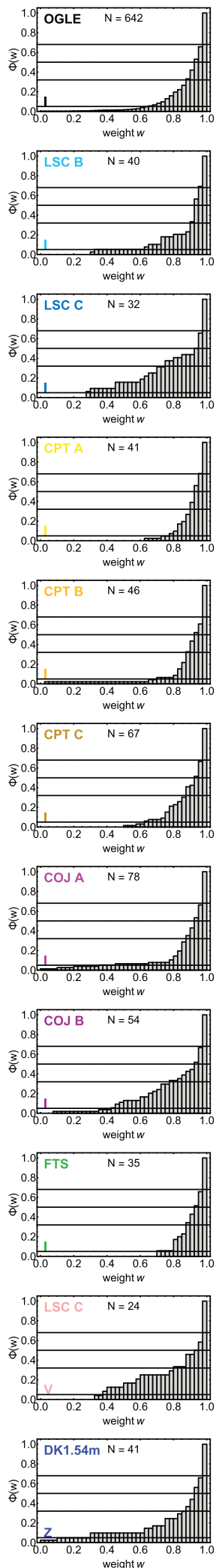

$u_{0}>0$
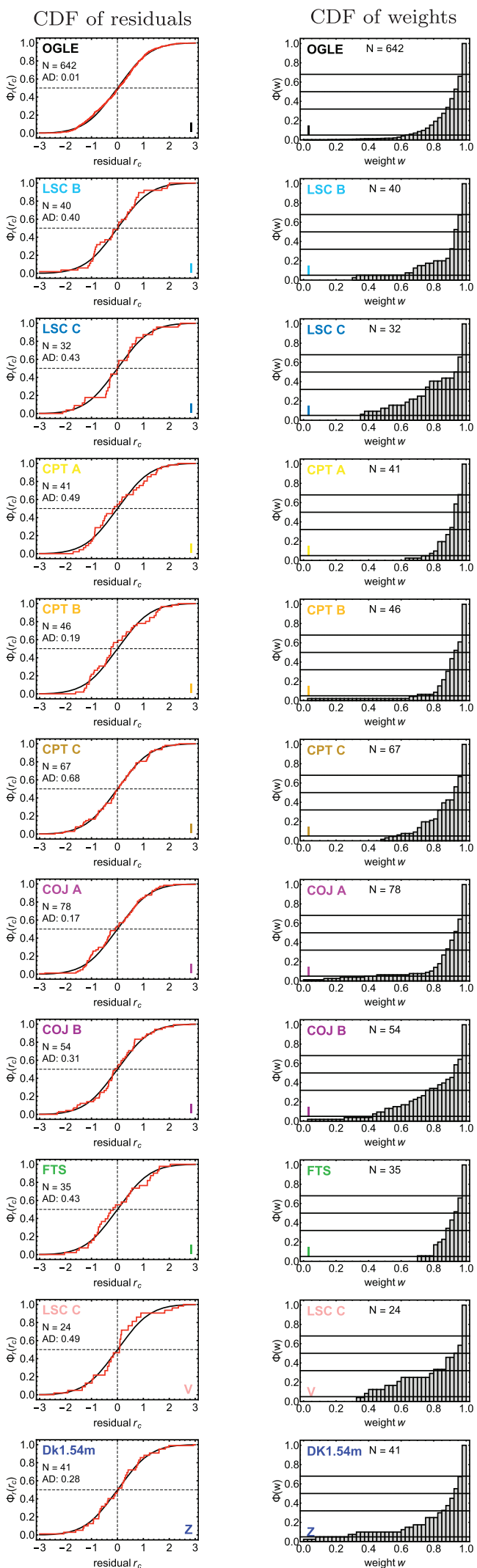

Figure 2. Weighted CDFs of the standardised residuals and CDF of data weights for the various off-peak data sets using the described robust-fitting procedure with single-lens point-source parallax models (see Tables 3 and 4) for $u_{0}<0$ and $u_{0}>0$, respectively. The distribution of the standardised residuals is compared to a standard Gaussian distribution, quoting the $p$-value of an AD test (Anderson \& Darling 1952). For the distribution of weights, cumulative probabilities of 5 per cent, 32 per cent, 50 per cent, and 68 per cent are indicated. 

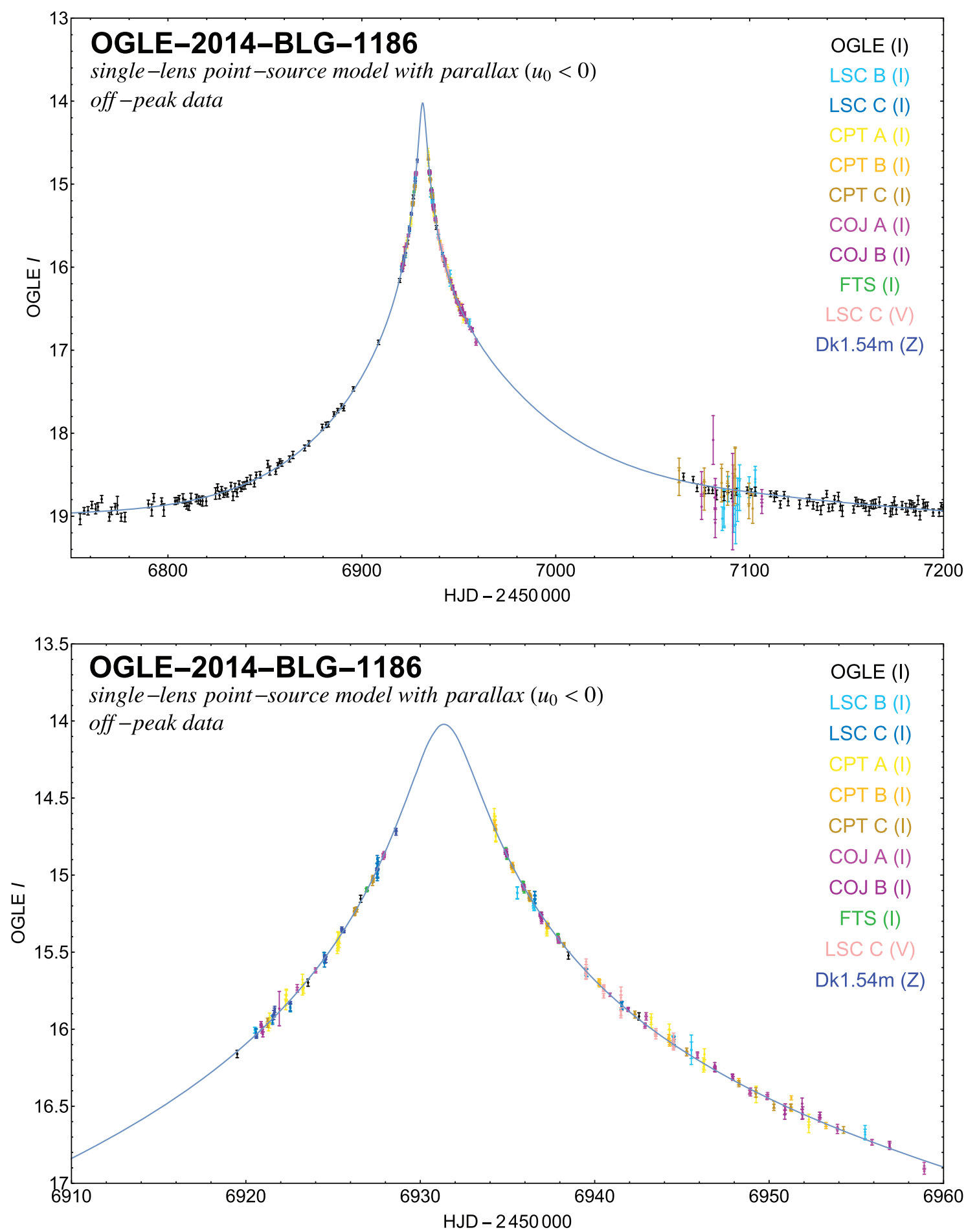

Figure 3. Acquired off-peak data on event OGLE-2014-BLG-1186 with the various telescopes together with a model light curve that assumes an isolated single lens as well as a point-like source and accounts for the annual parallax, where $u_{0}<0$ (see Table 3 ). The error bars displayed include a systematic error $s_{0}$ and scaling factor $\kappa$, as listed in Table 2 and determined with respect to the adopted model.

arising for binary (and multiple) lenses are characterised by the track of the source relative to the caustics, providing a type classification (Liebig et al. 2015).

The possible topologies of caustics are the same for all binary lenses (Erdl \& Schneider 1993), discriminated by the separation parameter $d$ for any given mass ratio $q$. For small mass ratios $q$, the intermediate topology with a single caustic curve with six cusps, occupies only a small range near $d \sim 1$, essentially leaving a close-binary $(d<1)$ and a wide-binary $(d>1)$ case (Griest \& Safizadeh 1998; Dominik 1999). In both of these cases, one finds a 'central caustic' around the centre of mass of the binary (i.e. factually near the host star for a star-planet system), which has two cusps along the binary axis, and a further two cusps symmetrically above and below. As $q \rightarrow 0$, the central caustics for pairs of close- 

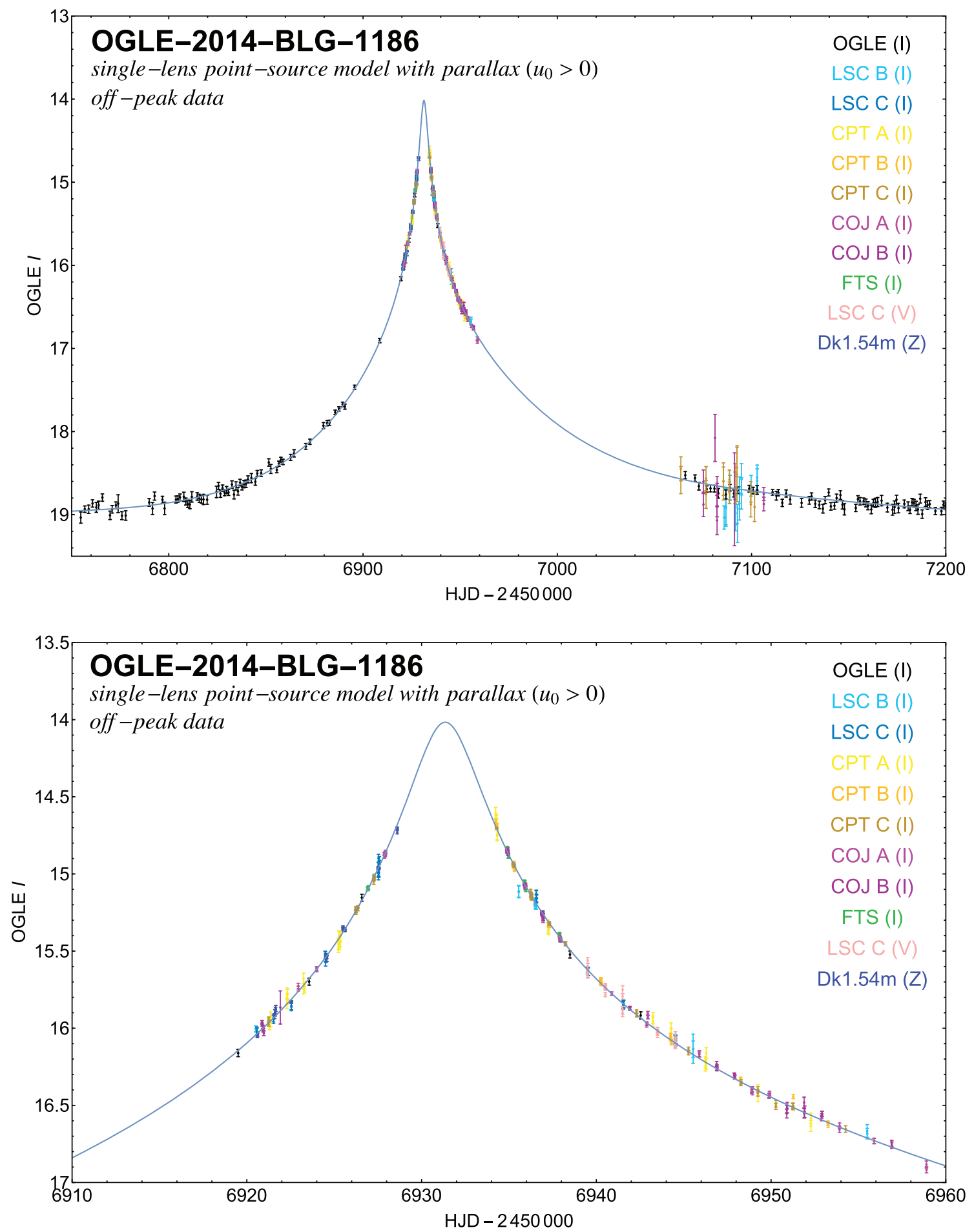

Figure 4. Acquired off-peak data on event OGLE-2014-BLG-1186 with the various telescopes together with a model light curve that assumes an isolated single lens as well as a point-like source and accounts for the annual parallax, similar to Fig. 3, but now for $u_{0}>0$ (see Table 4). The error bars displayed include a systematic error $s_{0}$ and scaling factor $\kappa$, as listed in Table 2, and determined with respect to the adopted model.

and wide-binary models with $d \leftrightarrow d^{-1}$ become identical, which causes a model ambiguity. Moreover, near a location that has an image under gravitational lensing by the star at the position of the planet, one finds 'planetary caustics'. In the case of a wide binary, there is a single diamond-shaped caustic with four cusps (two on the star-planet axis, and two above and below), whereas a close binary has two off-axis triangular-shaped caustics with three cusps each, where the longest side is close to parallel to the star-planet axis.

The magnification function $A[\boldsymbol{u}(t ; \boldsymbol{p})]$ for a binary lens, where $\boldsymbol{p}=\left(t_{0}, u_{0}, t_{E}, d, q, \alpha\right)$, neglecting the finite extent of the source star, is no longer an analytic function, but can be numerically evaluated by solving a fifth-order complex polynomial for the image positions (Witt \& Mao 1995; Skowron \& Gould 2012). 

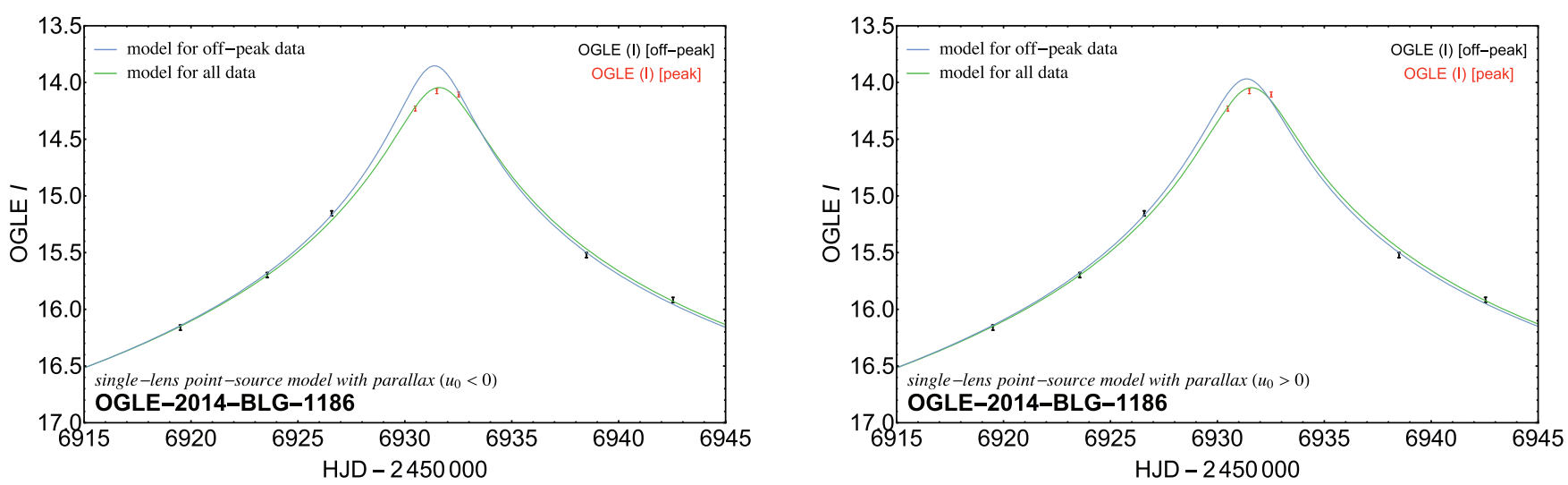

Figure 5. Single-lens point-source models with parallax fitted to OGLE data only, using either the off-peak data only (blue) or the full data set (green). The OGLE data do not obviously hint at an anomaly in event OGLE-2014-BLG-1186.

With the parameters $\left(t_{0}, u_{0}, t_{\mathrm{E}}, \pi_{\mathrm{E}, \mathrm{N}}, \pi_{\mathrm{E}, \mathrm{E}}\right)$ already being reasonably well determined from the off-peak data, we searched the complementary parameter sub-space $(d, q, \alpha)$, characterizing the lens binarity, for viable models incorporating the peak data. In fact, for fixed $\left(t_{0}, u_{0}, t_{\mathrm{E}}, \pi_{\mathrm{E}, \mathrm{N}}, \pi_{\mathrm{E}, \mathrm{E}}\right)$ and the adopted scaling of error bars (according to Table 2), we evaluated $\chi^{2}$ for a dense grid of ( $d$, $q, \alpha)$ for the peak data, just adjusting the baseline fluxes $F_{\text {base }}^{[j]}$ and the blend ratios $g^{[j]}$, so that $\chi^{2}$ is minimised. The resulting $\chi^{2}$ maps for the both cases $u_{0}<0$ and $u_{0}>0$ are shown in Fig. 7 .

Moreover, the binary-lens parameter space can be constrained straightforwardly from the morphology of the light curve. While we find an impact parameter $u_{0}<0.01$, the observed light curve does not exhibit any strong features arising from the source passing over a caustic. This immediately rules out any configuration with an intermediate caustic, while the size of the central caustic for a close or wide binary is restricted by the small impact parameter. Moreover, the shape of the anomaly over the peak suggests that the source first reaches a closest approach to the central caustic, producing the first (main) peak, and then passes close to one of the cusps of the central caustic, producing the further second peak. As illustrated in Fig. 8, this leaves us with only three options for the angle of the source trajectory with respect to the binary axis for each $u_{0}<0$ and $u_{0}>0$, which are identifiable as $\chi^{2}$ valleys in Fig. 7. Namely, the second peak can arise from the source passing near the cusp on the binary axis at the 'pointy end' towards the secondary (type 1), or the source passing near the off-axis cusp, with the trajectory either close to perpendicular to the binary axis (type 2) or close to parallel to the binary axis (type 3 ). The acquired data rule out configurations for which the source trajectory gets near the cusp on the binary axis that is opposite the secondary, because such would hit the caustic near at least one of the off-axis cusps. The $\chi^{2}$ maps (Fig. 7) also explicitly reveal the ambiguity $d \leftrightarrow d^{-1}$ between close- and wide-binary models for small mass ratios $q$.

\subsubsection{The only viable binary-lens models and parameter ambiguities}

With our $\chi^{2}$ maps for $(d, q, \alpha)$ and our further assessment of possible configurations, viable models must reside within a local minimum that corresponds to one of the 12 options given by $u_{0}$ $<0$ or $u_{0}>0, d<1$ or $d>1$, and one of the three trajectory types shown in Fig. 8. Local $\chi^{2}$ optimisation of the full parameter space $\left(t_{0}, t_{\mathrm{E}}, u_{0}, \pi_{\mathrm{E}, \mathrm{N}}, \pi_{\mathrm{E}, \mathrm{E}}, d, q, \alpha, \boldsymbol{F}_{\text {base }}, \boldsymbol{g}\right)$ for all data shows that type 2 and 3 trajectories cannot reasonably account for the data, given that best-fitting model light curves are clearly visually off the data, leaving us with the four models listed in Tables 3 and 4, whose light curves are shown together with the peak data in Fig. 6, and no further possible options. Type 2 and 3 trajectories fail on the requirement that in order to match the data, the impact parameter $u_{0}$, the trajectory angle $\alpha$, and the time-scale $t_{\mathrm{E}}$ must meet the size of the caustic and the time interval between the two observed peaks. We find that the values $\left(t_{\mathrm{E}}, u_{0}, \pi_{\mathrm{E}, \mathrm{N}}, \pi_{\mathrm{E}, \mathrm{E}}\right)$ are essentially identical to what we estimated from the off-peak data, passing the check of robustness and consistency of our approach.

Visual inspection of the model light curves and the peak data (as shown in Fig. 6) reveals a few low-level discrepancies: (1) most significantly, over the second peak, the slope of the model light curve is not in agreement with what two data sets (COJ B and FTS) independently suggest, (2) between the two peaks, the model favours the LSC B and LSC C data, while substantially disfavouring the OGLE data, (3) the CPT C and OGLE data over the main peak are systematically above the model light curve, (4) the OGLE, LSC B, LSC B, and FTS data just ahead of the main peak are all below the model light curve, (5) the FTS data just after the first peak are all above the model light curve.

At this stage, we looked into the effect of the finite size of the source star on the light curve, which becomes significant for strong differential magnification with substantial second derivatives. It can be described by means of a dimensionless parameter $\rho_{\star}$, where $\rho_{\star} \theta_{\mathrm{E}}$ is the angular source radius, and to first order the star can be approximated as being uniformly bright. For the evaluation of the magnification for given model parameters, we have adopted a contour integration algorithm (Dominik 1993; Gould \& Gaucherel 1997; Dominik 1998c) improved with parabolic correction, optimal sampling and accurate error estimates, as described in detail by Bozza (2010).

Considering the finite source star size with our binary-lens pointsource parallax models, we find that the major differences arise over the second peak, which deforms into a shoulder at around $\rho_{\star} \sim 2 \times 10^{-3}$, whereas a light curve for $5 \times 10^{-4}$ is rather close to the point-source case. We apply the PYLIMA software suite (Bachelet et al. 2017), using differential evolution, to find the binary-lens finite-source parallax models, whose parameters are given in Table 5. For these models, we also show the binary-lens caustics and the respective source trajectory in Fig. 9.

The model light curves over the peak region are shown in Fig. 10, which do not exhibit any visible differences amongst the four ambiguous models. Comparing the models with finite source size 

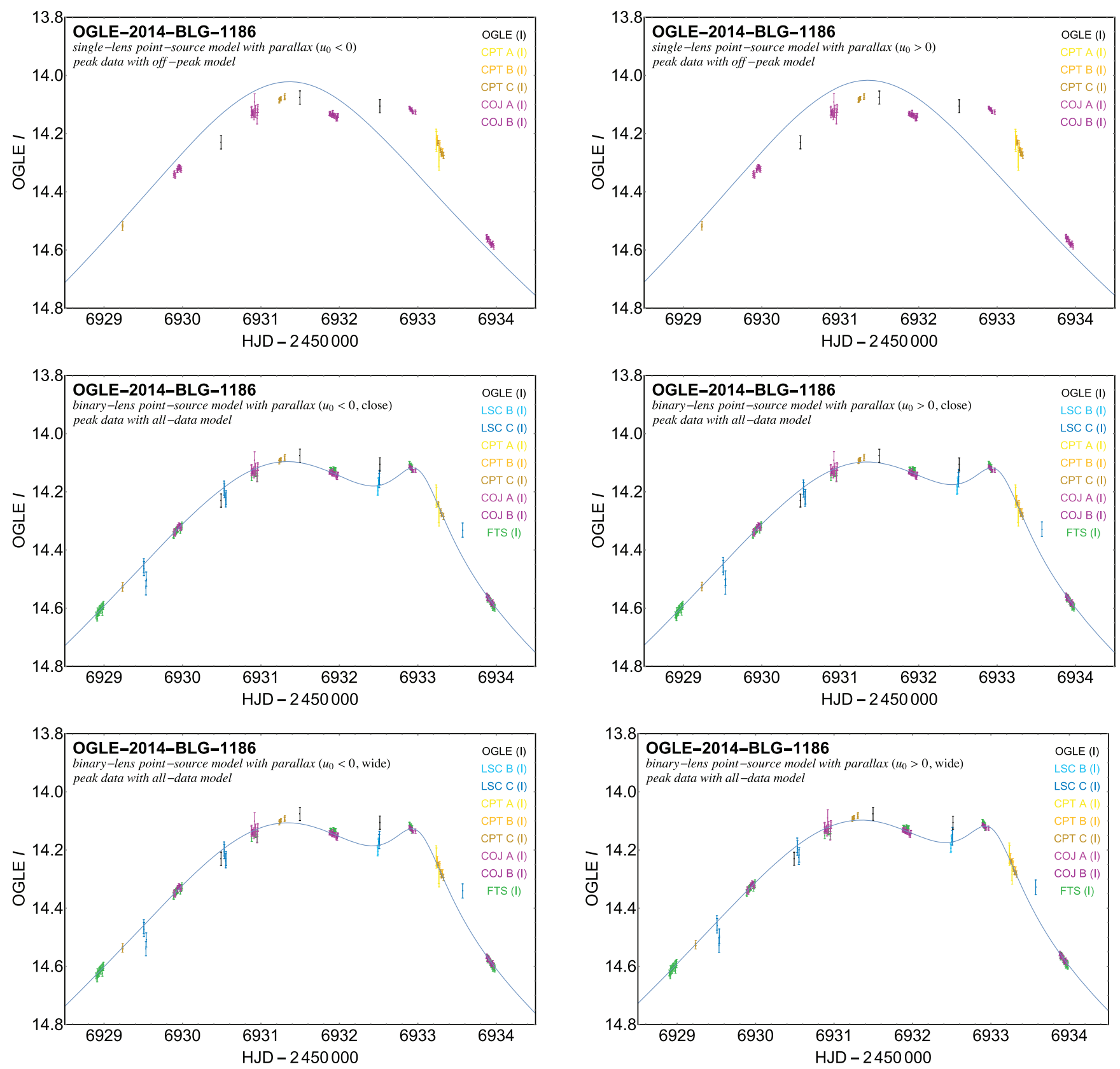

Figure 6. Peak anomaly of microlensing event OGLE-2014-BLG-1186. The upper panels show the single-lens point-source parallax models to the off-peak data for $u_{0}<0$ or $u_{0}>0$, respectively (c.f. Tables 3 and 4) with the peak data, aligned according to the baseline fluxes $\boldsymbol{F}_{\text {base }}$ and blend ratios $\boldsymbol{g}$ suggested by the models for the off-peak data. Only those data sets for which these parameters could be well determined are shown. These align very well, giving a clear and consistent picture of the anomaly over the peak. The middle and lower panels compare binary-lens point-source parallax models for the four cases $u_{0}<$ 0 or $u_{0}>0$ as well as $d<1$ or $d>1$ (c.f. Tables 3 and 4) with the acquired peak data, showing that such models can account for the major features of the double-peak light curve, but most notably do not match the slope indicated by the COJ A and FTS data over the second peak.

with those with a point-like source star, we find that considering the finite size of the star successfully removes the previously found problem with the wrong slope over the second peak. Moreover, the discrepancy of the OGLE point just before the second peak is reduced. However, the finite size of the source star has little effect on the first peak. We have neglected any orbital motion or effects from any further massive bodies within the lens system. These would cause only quite small changes to the photometric light curve, at a level potentially comparable with systematic noise, preventing a reliable measurement of the underlying parameters. Given that we cannot do any better within the adopted model, we regard the model parameters as robust.

The fourfold ambiguity corresponds to close or wide binaries $(d<1$ or $d>1)$, as well as $u_{0}<0$ or $u_{0}>0$, where $\left(u_{0}, \alpha, \pi_{\mathrm{E}}\right) \leftrightarrow\left(-u_{0},-\alpha, \pi_{\mathrm{E}}\right)$. We explicitly note that we do not find any of the parallax ambiguities described by Skowron et al. (2011), in particular not $\left(u_{0}, \alpha, \boldsymbol{\pi}_{\mathrm{E}}\right) \leftrightarrow\left(-u_{0},-\alpha,-\boldsymbol{\pi}_{\mathrm{E}}\right)$, which holds if the parallax affects the microlensing light curve mainly by a local effective acceleration near the peak. In contrast, we find this acceleration to be small and of opposite sign in our $u_{0}<0$ and 

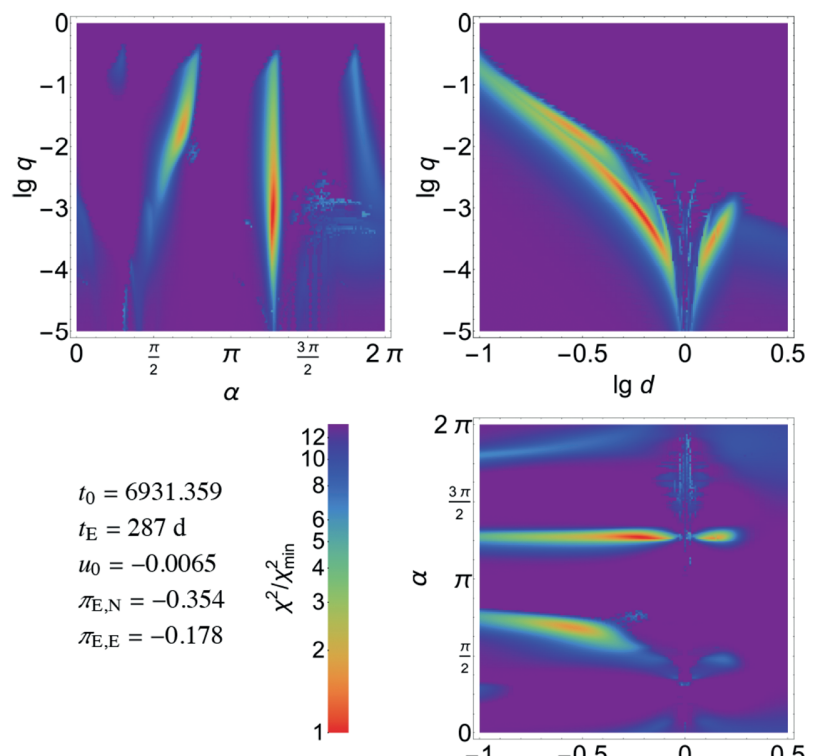

OGLE-2014-BLG-1186

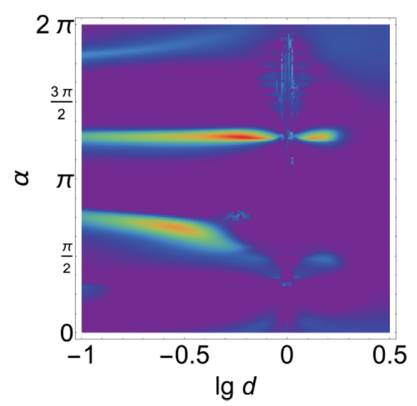

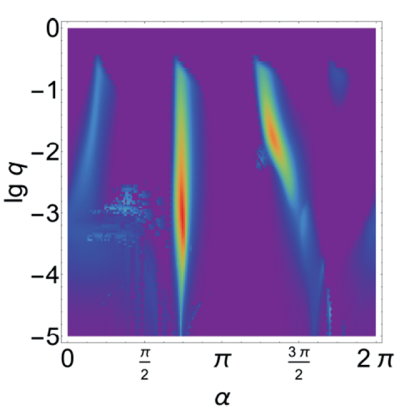
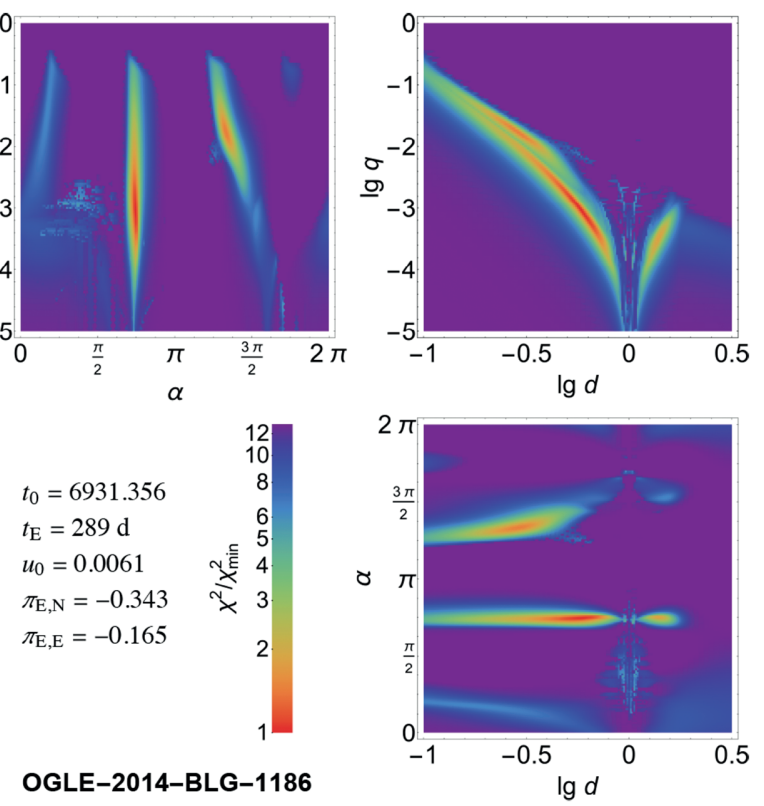

Figure 7. Exploration of binary-lens parameter space. Colour-coded values of $\chi^{2}$ for the peak data as a function of the binary-lens parameters $(d, q, \alpha)$ as three diagrams of $\chi^{2}$ as a function of $(\lg q, \alpha),(\lg d, \lg q)$, and $(\lg d, \alpha)$, where each reported value of $\chi^{2}$ corresponds to the minimum over the remaining third parameter. These diagrams have been positioned so that all three parameters corresponding to local minima can readily be identified. The parameters $\left(t_{0}, t_{\mathrm{E}}, u_{0}\right.$, $\pi_{\mathrm{E}, \mathrm{N}}, \pi_{\mathrm{E}, \mathrm{E}}$ ) have been kept fixed to values suggested by single-lens point-source parallax models for the off-peak data for $u_{0}<0$ or $u_{0}>0$, respectively (c.f. Tables 3 and 4), and only the baseline fluxes $F_{\text {base }}^{[j]}$ and the blend ratios $g^{[j]}$ have been adjusted for each $(d, q, \alpha)$ in order to minimize $\chi^{2}$. The colour scale has been normalized, so that the absolute minimum corresponds to the red end, while a single lens or any configuration with a larger $\chi^{2}$ corresponds to the purple end. While for small mass ratios $q$, one finds an ambiguity $d \leftrightarrow d^{-1}$ for the separation parameter, the valleys distinguished by the trajectory angle $\alpha$ correspond to the three possible types for the specific morphology observed in the light curve, illustrated in Fig. 8 .

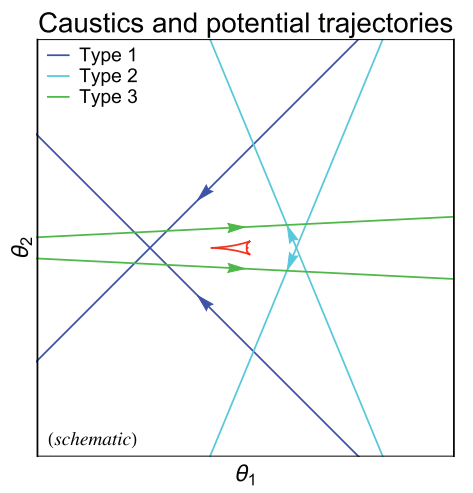

Figure 8. Schematic illustration of the three possible types of trajectories that can produce a light curve with the observed features. These are to arise from a very close approach of the source to a central caustic (first peak), with a subsequent approach to one of its cusps producing the second peak. The small impact parameter $u_{0}$ rules out intermediate topologies. For each type of trajectory, there are two realisations for the impact parameter $u_{0}$ and trajectory angle $\alpha$, distinguished by $\left(u_{0}, \alpha\right) \leftrightarrow\left(-u_{0},-\alpha\right)$. The three different types are clearly seen in the $\chi^{2}$ plots exploring the binary-lens parameter space, as shown in Fig. 7.

$u_{0}>0$ models, while the parallax results in a substantial distortion of the wings of the light curve over 4 yr. In fact, Fig. 11 illustrates the effect of parallax in the 2013 and early 2014 data, as well as in the late 2015 and 2016 data.

For the $u_{0}<0$ wide-binary model, the source trajectory gets close to the planetary caustic, resulting in a further small feature (see Fig. 12), most of it falling into a gap of data coverage. For this reason, this model appears to stand out slightly from the others with respect to the parameters. However, the details of the approach to the planetary caustic depend on the orbital motion which is present but cannot be reliably determined. Therefore, this potential feature does not provide us with an opportunity to distinguish between the four models.

\subsection{Binary-source models}

Double-peaked microlensing events can also arise if the source rather than the lens object is a binary (Griest \& Hu 1992). We should therefore carefully consider a binary-lens interpretation of the observed data as an alternative to our binary-lens models.

The gravitational magnification of a binary source is straightforwardly given as the linear superposition of the magnification of its components, i.e.

$$
\begin{aligned}
A_{\mathrm{BS}}\left(\boldsymbol{u}^{(1)}, \boldsymbol{u}^{(2)}, \omega_{\lambda}, \boldsymbol{\pi}_{\mathrm{E}}\right)= & \left(1-\omega_{\lambda}\right) A\left[u\left(t, t_{0}^{(1)}, u_{0}^{(1)}, t_{\mathrm{E}}, \boldsymbol{\pi}_{\mathrm{E}}\right)\right] \\
& +\omega_{\lambda} A\left[u\left(t, t_{0}^{(2)}, u_{0}^{(2)}, t_{\mathrm{E}}, \boldsymbol{\pi}_{\mathrm{E}}\right)\right],
\end{aligned}
$$

with $u\left(t ; t_{0}, u_{0}, t_{\mathrm{E}}, \pi_{\mathrm{E}}\right)$ given by equation (24) and

$\omega_{\lambda}=\frac{L_{2, \lambda}}{L_{1, \lambda}+L_{2, \lambda}}$

being the luminosity offset ratio depending on the wavelength filter used, while $L_{1, \lambda}$ and $L_{2, \lambda}$ denote the luminosities of the two source stars. For a uniformly bright source of angular radius $\rho_{\star} \theta_{\mathrm{E}}$, the magnification $A\left(u, \rho_{\star}\right)$ due to a point-mass lens can be computed efficiently in terms of complete elliptic integrals (Witt \& Mao 1994).

Given that all data acquired over the peak of event OGLE-2014BLG-1186 are in $I$ band and the effect of binarity on the photometric light curve is negligible for other epochs, we describe single-lens 
Table 5. Parameters of the four successful binary-lens finite-source parallax models, distinguished by the side on which the source passes relative to the lens near the peak $\left(u_{0}<0\right.$ or $\left.u_{0}>0\right)$, and whether the binary lens is in a 'close' $(d<1)$ or 'wide' $(d>1)$ configuration. We adopted error bars arising from a scaling based on the off-peak data, and obtained a simple maximum-likelihood (ML) estimate on all data. The respective value of $\chi^{2}$ is reported for reference.

\begin{tabular}{|c|c|c|c|c|}
\hline \multirow{6}{*}{$\begin{array}{l}\text { Model } \\
\text { Data selection } \\
\text { Data sets } \\
\text { Data scaling } \\
\text { Minimisation } \\
\text { Option }\end{array}$} & \multirow{2}{*}{\multicolumn{4}{|c|}{ Binary, parallax, finite source }} \\
\hline & & & & \\
\hline & \multicolumn{4}{|c|}{ All } \\
\hline & $u_{0}<0$ off-peak & $u_{0}<0$ off-peak & $u_{0}>0$ off-peak & $u_{0}>0$ off-peak \\
\hline & \multicolumn{4}{|c|}{ 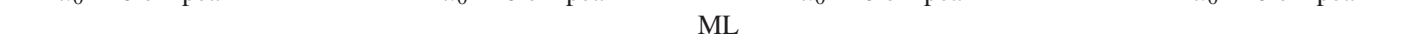 } \\
\hline & $u_{0}<0$, close & $u_{0}<0$, wide & $u_{0}>0$, close & $u_{0}>0$, wide \\
\hline$t_{0}$ & $6931.455 \pm 0.004$ & $6931.495 \pm 0.004$ & $6931.421 \pm 0.004$ & $6931.479 \pm 0.004$ \\
\hline$t_{E}[\mathrm{~d}]$ & $271 \pm 19$ & $237 \pm 10$ & $277 \pm 19$ & $270 \pm 18$ \\
\hline$\pi_{\mathrm{E}, \mathrm{E}}$ & $-0.191 \pm 0.010$ & $-0.205 \pm 0.009$ & $-0.176 \pm 0.009$ & $-0.179 \pm 0.009$ \\
\hline$d$ & $0.734 \pm 0.008$ & $1.366 \pm 0.012$ & $0.702 \pm 0.009$ & $1.439 \pm 0.015$ \\
\hline$q$ & $(3.4 \pm 0.3) \times 10^{-4}$ & $(3.8 \pm 0.2) \times 10^{-4}$ & $(4.1 \pm 0.3) \times 10^{-4}$ & $(4.2 \pm 0.3) \times 10^{-4}$ \\
\hline$\alpha$ & $4.045 \pm 0.004$ & $4.046 \pm 0.004$ & $2.312 \pm 0.005$ & $2.311 \pm 0.004$ \\
\hline$\rho_{\star}$ & $(10.1 \pm 1.6) \times 10^{-4}$ & $(12.3 \pm 1.5) \times 10^{-4}$ & $(9.7 \pm 1.8) \times 10^{-4}$ & $(9.6 \pm 1.5) \times 10^{-4}$ \\
\hline
\end{tabular}
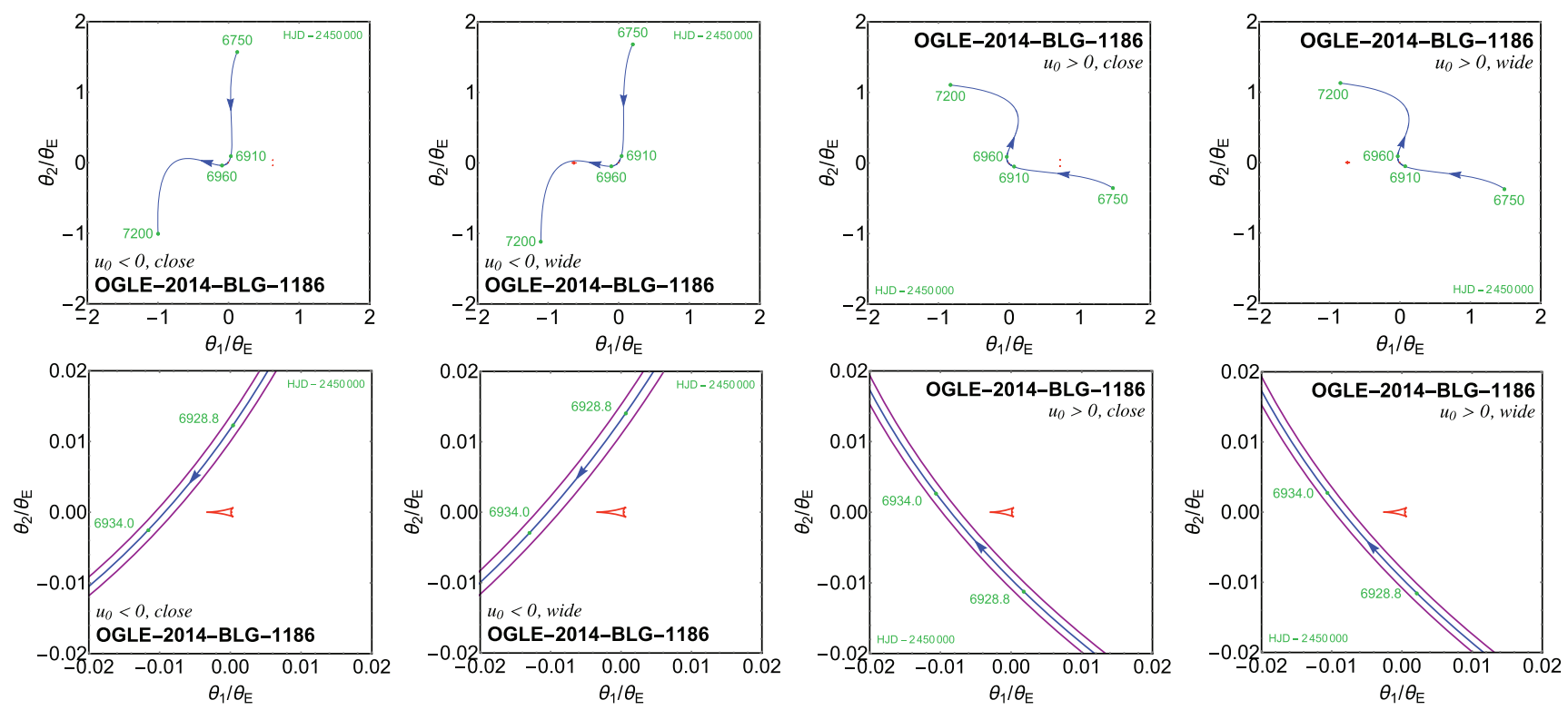

Figure 9. Binary-lens caustics (in red) and source trajectory (in blue, indicating the finite source size by magenta lines) for the four binary-lens finite-source parallax models whose respective parameters are listed in Table 5. Specific epochs are marked by green dots, and the direction of the source along the trajectory is indicated by arrows. The fourfold model ambiguity corresponds to solution with $u_{0}<0$ or $u_{0}>0$ on one hand, as well as close or wide binaries $(d<1$ or $d>1)$ on the other hand. With the sign of the impact parameter $u_{0}$, the sign of the trajectory angle $\alpha$ gets inverted as well $(\alpha \leftrightarrow-\alpha$ or $\alpha \leftrightarrow 2 \pi-\alpha)$. The parallax effect is prominent in the wings of the light curve, which determine the parallax parameters $\left(\pi_{\mathrm{E}, \mathrm{N}}, \pi_{\mathrm{E}, \mathrm{E}}\right)$, while the local effective acceleration of the source near the peak is small, with opposite curvature of the source trajectory for the $u_{0}<0$ and $u_{0}>0$ cases. For the $u_{0}<0$ wide-binary model, the source trajectory gets close to the planetary caustic, resulting in a further feature (see Fig. 12).

binary-finite-source models with annual parallax by the parameter vector $\boldsymbol{p}=\left(t_{0}^{(1)}, t_{0}^{(2)}, t_{\mathrm{E}}, u_{0}^{(1)}, u_{0}^{(2)}, \pi_{\mathrm{E}, \mathrm{N}}, \pi_{\mathrm{E}, \mathrm{E}}, \omega_{I}, \rho_{\star}^{(1)}, \rho_{\star}^{(2)}\right)$, explicitly defining the reference epoch for parallax $t_{0}$, equations (16) and (19), to refer to the $I$-band photocentre

$t_{0} \equiv\left(1-\omega_{I}\right) t_{0}^{(1)}+\omega_{I} t_{0}^{(2)}$.

This leads to the four sets of best-fitting model parameters listed in Table 6, which are distinguished by all combinations of the respective signs of $u_{0}^{(1)}$ and $u_{0}^{(2)}$. In the absence of significant parallax effects, binary-source models become blind to whether the two source stars are on the same side of the effective lens trajectory, i.e. $u_{0}^{(1)} u_{0}^{(2)}>0$ ('cis' configuration), or on opposite sides, i.e. $u_{0}^{(1)} u_{0}^{(2)}<$
0 ('trans' configuration) (Dominik \& Hirshfeld 1996, appendix C). We find the two source stars being separated by an angle $\lambda \theta_{\mathrm{E}}$, where

$\lambda=\sqrt{\left(\frac{t_{0}^{(2)}-t_{0}^{(1)}}{t_{\mathrm{E}}}\right)^{2}+\left(u_{0}^{(2)}-u_{0}^{(1)}\right)^{2}}$,

which for our models evaluates to

$$
\begin{aligned}
\lambda^{\text {cis }} & =0.0087 \pm 0.0008, \\
\lambda^{\text {trans }} & =0.0107 \pm 0.0009 .
\end{aligned}
$$



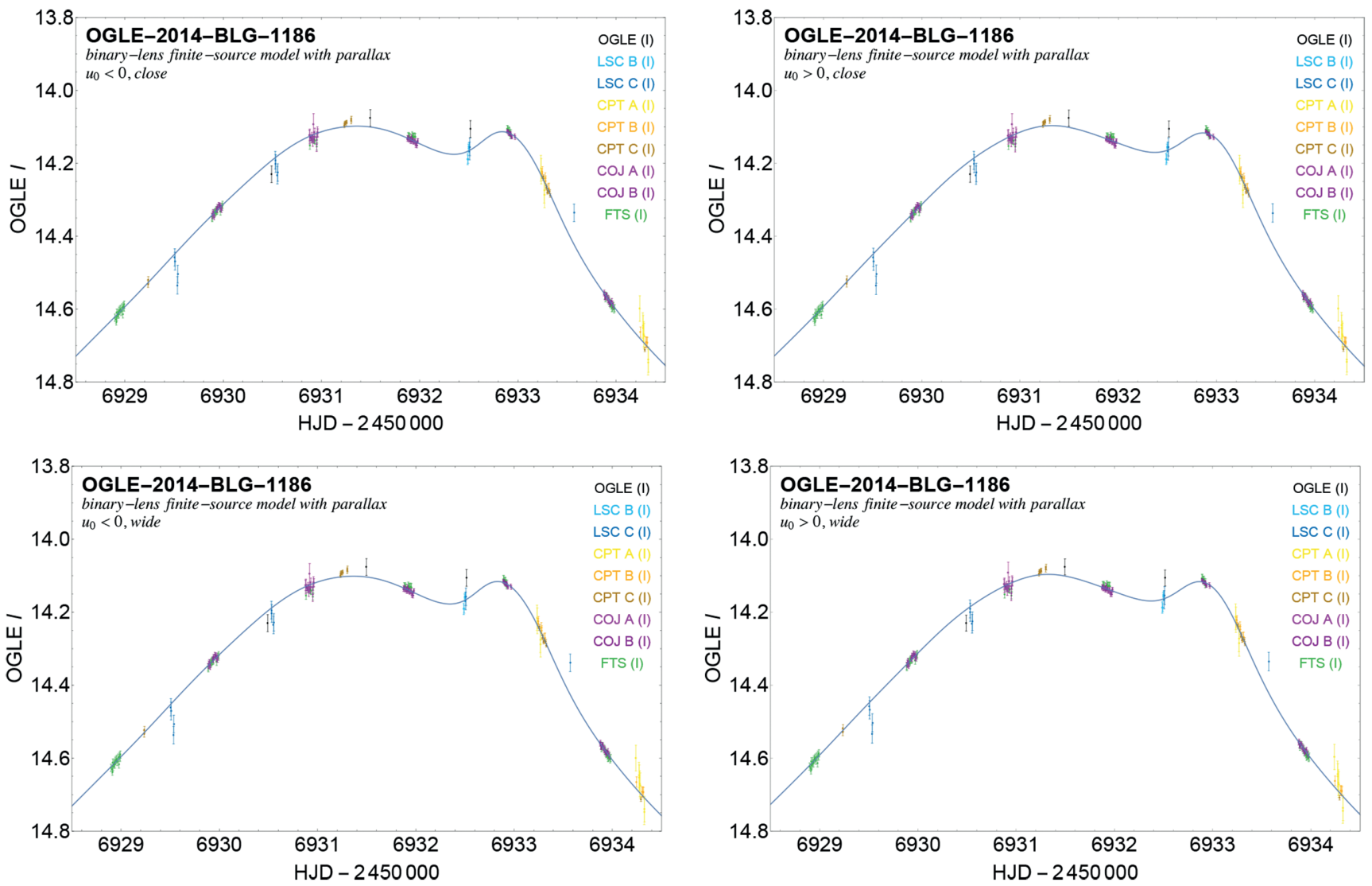

Figure 10. Photometric data in peak region along with the four different binary-lens finite-source models with parallax whose parameters are listed in Table 5. The different underlying geometries produce pretty much the same light curve, which is moreover quite close to those found with the point-source models (Tables 3 and 4, Fig. 6), the most visible difference being the slope through the data points near the second peak (close to HJD $-2450000=6933$ ).
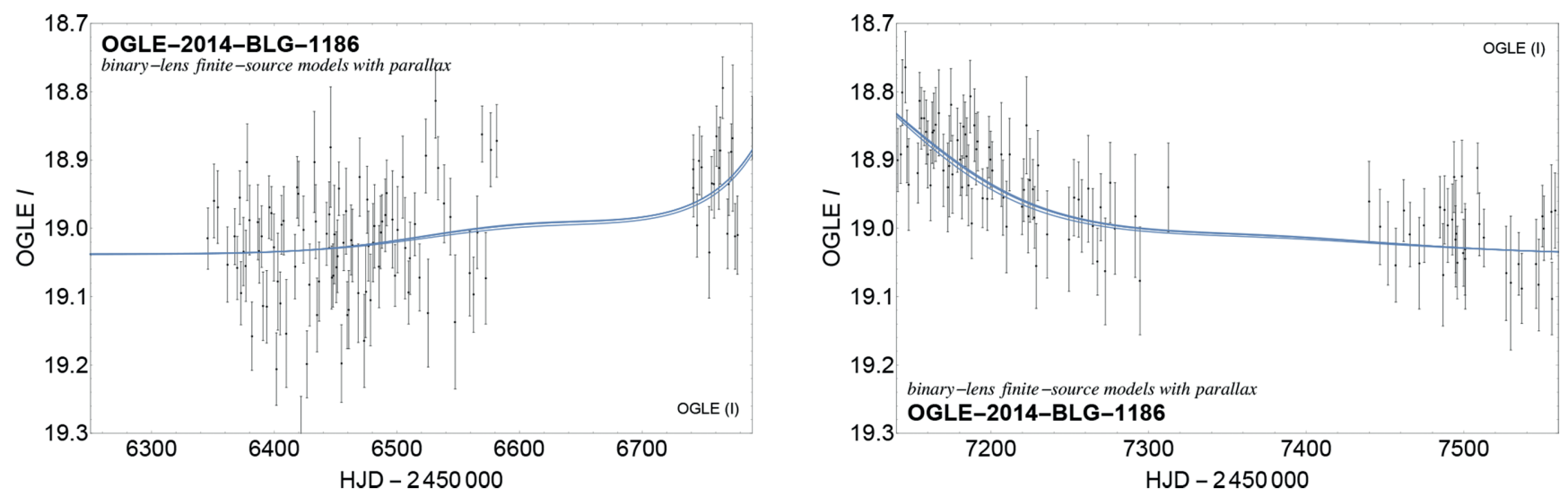

Figure 11. The effect of annual parallax on the light curve in early and late event phases close to the baseline magnitude, with light curves corresponding to each of the finite-source binary-lens parallax models listed in Table 5, is shown. The left-hand panel shows data from 2013 and 2014 , whereas the right-hand panel shows data from 2015 and 2016. The event OGLE-2014-BLG-1186 was evidently above its baseline magnitude over the course of 4 yr.

Our binary-source models involve a brighter larger source star dominating the (earlier) main peak, while the (later) secondary peak is due to a fainter smaller source star that passes the lens star at a smaller minimal separation.

The respective light curves are shown in Fig. 13, which are apparently hardly distinguishable from those corresponding to the identified viable binary-lens models (Fig. 10). In particular, the difference between the two presented models is not larger than the differences between model and data. If we were to trust our data at that level (excluding that any residuals are due to systematic uncertainties), we would need to reject both models. If we accept that there are systematic uncertainties at that level, we would need to accept both. We explicitly show the difference between the light curves of two of our binary-lens and binary-source models in 

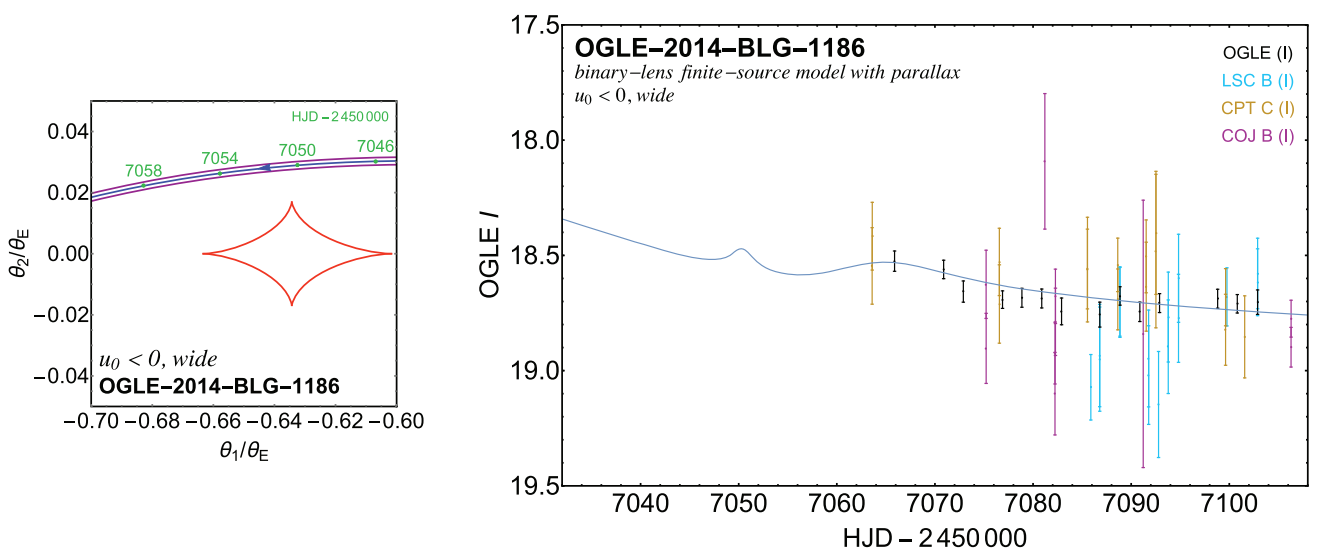

Figure 12. Bump in the light curve for the $u_{0}<0$ finite-source wide-binary model with parallax (c.f. Table 5), arising from the source approaching the vicinity of the planetary caustic. The left-hand panel shows the planetary caustic (in red) together with the effective source trajectory (in blue), with the source size indicated by the purple lines. Specific epochs are marked by green dots. The right panel shows the model light curve around the bump together with the acquired data. While there is a lack of photometric data around the epoch at which this feature shows, the neglected but existent orbital motion of the planet can alter it and make it essentially disappear. It is therefore unsuitable to provide a distinction between the four presented models.

Table 6. Parameters of four successful single-lens binary-finite-source parallax models, distinguished by the side on which each of the source stars passes relative to the lens near the peak. Given that all peak data have been acquired in $I$ band and the binarity does not significantly affect the light curve outside the peak region, we use a single luminosity offset ratio $\omega_{I}$ characteristic for $I$ band. We adopted error bars arising from a scaling based on the off-peak data, and obtained a simple maximum-likelihood (ML) estimate on all data. The respective value of $\chi^{2}$ is reported for reference.

\section{Model}

Data selection

Data sets

Data scaling

Minimisation

Option

$t_{0}^{(1)}$

$t_{0}^{(2)}$

$t_{E}[\mathrm{~d}]$

$u_{0}^{(1)}$

$u_{0}^{(2)}$

$\pi_{\mathrm{E}, \mathrm{N}}$

$\pi_{\mathrm{E}, \mathrm{E}}$

$\omega_{I}$

$\rho_{\star}^{(1)}$

$\rho_{\star}(2)$

$\chi^{2}$

Single lens, parallax, binary finite source

All

All

ML

$$
u_{0}>0 \text { off-peak } \quad u_{0}>0 \text { off-peak }
$$

$u_{0}^{(1)}<0, u_{0}^{(2)}>0$

$6931.229 \pm 0.007$

$6932.945 \pm 0.007$

$306 \pm 19$

$-0.0082 \pm 0.0005$

$0.00142 \pm 0.00008$

$-0.363 \pm 0.009$

$-0.178 \pm 0.008$

$0.040 \pm 0.002$

$(7.9 \pm 0.5) \times 10^{-3}$

$(1.6 \pm 0.1) \times 10^{-3}$

1715 $u_{0}^{(1)}>0, u_{0}^{(2)}>0$

$6931.234 \pm 0.007$

$6932.944 \pm 0.007$

$311 \pm 19$

$0.0075 \pm 0.0005$

$0.00142 \pm 0.00008$

$-0.347 \pm 0.009$

$-0.158 \pm 0.008$

$0.044 \pm 0.002$

$(7.3 \pm 0.5) \times 10^{-3}$

$(1.6 \pm 0.1) \times 10^{-3}$

1716 $u_{0}^{(1)}>0, u_{0}^{(2)}<0$

$6931.234 \pm 0.007$

$6932.987 \pm 0.007$

$311 \pm 19$

$0.0075 \pm 0.0005$

$-0.00116 \pm 0.00008$

$-0.347 \pm 0.009$

$-0.158 \pm 0.008$

$0.045 \pm 0.002$

$(7.3 \pm 0.5) \times 10^{-3}$

$(1.6 \pm 0.1) \times 10^{-3}$

1716
Fig. 14. Taking into account a difference in the blend ratio relative to the OGLE data, the difference between the models is almost always below 5 mmag, except for short epochs near the second peak that are not or poorly covered by data.

We continue our discussion of the viability of the binary-lens and binary-source models in Section 4.3 after having investigated what the inferred model parameters mean for the physical nature of the lens and source systems.

\section{INTERPRETATION}

\subsection{Lens binary}

Following the approach suggested by Albrow et al. (2000), we use the de-reddened colour $(V-I)_{0}$ and the brightness $I_{0}$ of the source star to estimate its angular radius $\theta_{\star}$. Exploiting the fact that
OGLE monitors its fields not only in $I$ but also more sparsely in $V$, we construct an instrumental colour-magnitude diagram (CMD), shown in Fig. 15. We find the source star at $(V-I, I)=(2.76 \pm 0.05$, $19.42 \pm 0.12$ ), where a major uncertainty arises from the blend ratio, where $I$ - and $V$-band blend ratios are strongly correlated with the event time-scale $t_{\mathrm{E}}$ and with each other. The centroid of the Galactic bulge red clump $(\mathrm{RC})$ is at $(V-I, I)_{\mathrm{GC}}=(3.19 \pm 0.01$, $17.06 \pm 0.02)$. According to Bensby et al. (2011) and Nataf et al. (2013), the de-reddened colour and brightness of the red clump are $(V-I, I)_{\mathrm{RC}, 0}=(1.06,14.62)$ for the Galactic longitude of the target $l \sim 5^{\circ}$. Consequently, we find for our source star $(V-I$, $I)_{0}=(0.63 \pm 0.05,16.98 \pm 0.12)$, indicative of an F-type dwarf or a G-type subgiant.

For such stars, Kervella \& Fouqué (2008) provide a direct empirical relation to estimate the angular source radius $\theta_{\star}$ from $V$ and Cousins $I$ measurements (matching the OGLE filters), 

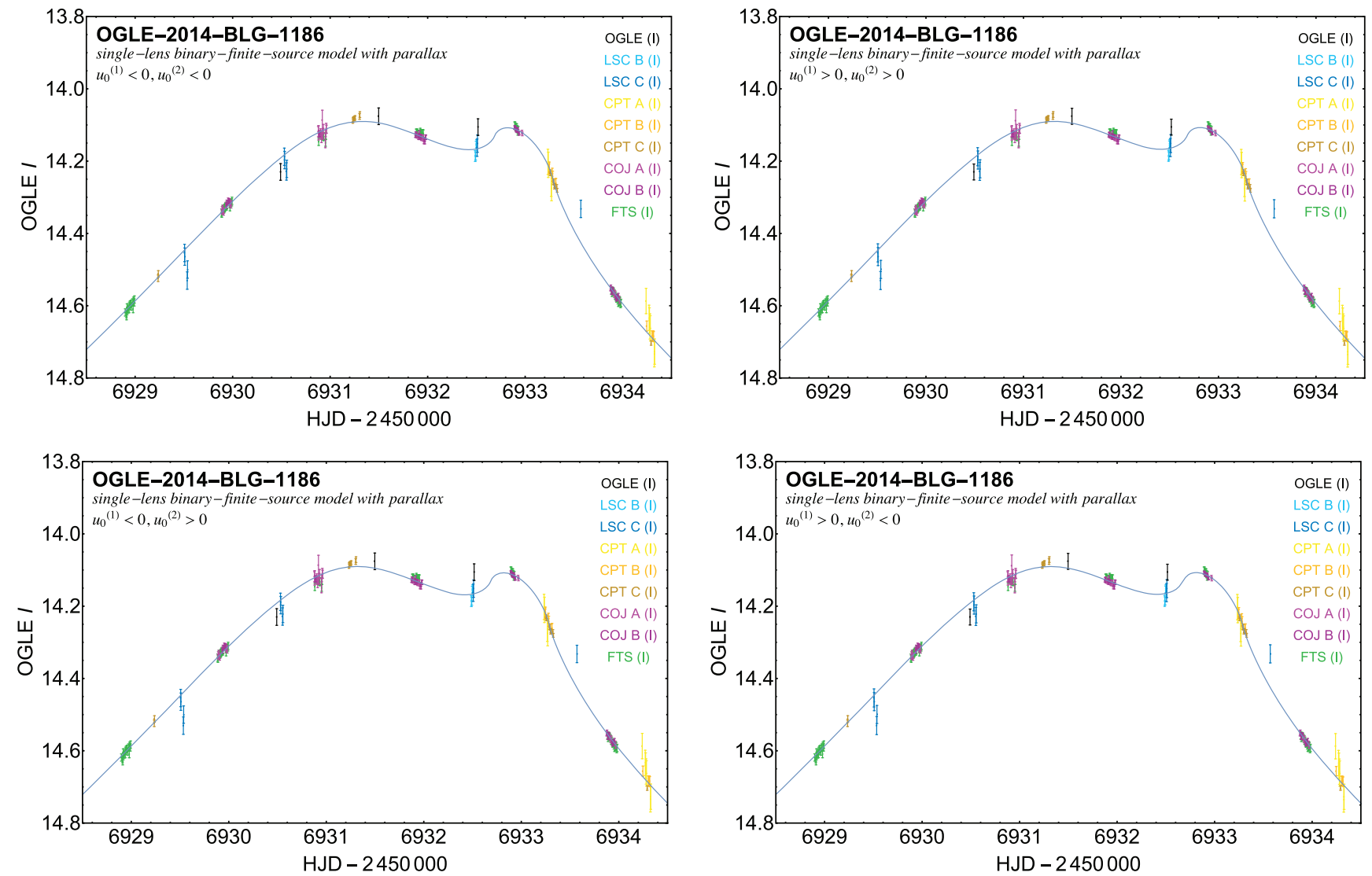

Figure 13. Photometric data covering the peak region along with the four single-lens binary-finite-source models with parallax whose parameters are listed in Table 6. These produce almost identical light curves, which moreover visibly differ from those produced by the binary-lens finite-source models (Table 5 and Fig. 10) only on the shape of the secondary peak during epochs not covered by data.

namely

$$
\begin{aligned}
\lg \left(\frac{2 \theta_{\star}}{1 \mathrm{mas}}\right)= & 0.4992+0.4895(V-I)_{0}-0.0657(V-I)_{0}^{2} \\
& -0.2 I_{0}
\end{aligned}
$$

so that we obtain

$\theta_{\star}=(1.21 \pm 0.11) \mu \mathrm{as}$,

including a typical uncertainty of 5.6 percent for the empirical relation. With the assumption of the source star being close to the Galactic bulge,

$D_{\mathrm{S}}=(8.5 \pm 2.0) \mathrm{kpc}$,

we estimate the physical radius of the source star $R_{\star}=D_{\mathrm{S}} \theta_{\star}$ to be

$R_{\star}=(2.2 \pm 0.6) R_{\odot}$.

From our models, we find the parallax parameter $\pi_{\mathrm{E}}=\pi_{\mathrm{LS}} / \theta_{\mathrm{E}}$ and the source size parameter $\rho_{\star}=\theta_{\star} / \theta_{\mathrm{E}}$ as

$\pi_{\mathrm{E}}=0.41 \pm 0.01$,

$\rho_{\star}=(10.4 \pm 1.6) \times 10^{-4}$,

while equation (4) gives the total mass as

$M=\frac{c^{2}}{4 G}(1 \mathrm{AU}) \frac{\theta_{\star}}{\pi_{\mathrm{E}} \rho_{\star}}$, which evaluates to

$M=(0.35 \pm 0.06) M_{\odot}$.

With the mass ratio

$q=(3.9 \pm 0.3) \times 10^{-4}$

we then find the mass of the planet as

$M_{2}=(45 \pm 9) M_{\oplus}$,

about 3 times the mass of Neptune or about half the mass of Saturn. The uncertainty in the mass measurement is dominated by the uncertainty in the source size parameter $\rho_{\star}$ (about 15 per cent).

From $\theta_{\star}$ and $\rho \star=\theta_{\star} / \theta_{\mathrm{E}}$, we obtain the angular Einstein radius as

$\theta_{\mathrm{E}}=(1.2 \pm 0.2) \mathrm{mas}$,

and with the event time-scale

$t_{\mathrm{E}}=(264 \pm 17) \mathrm{d}$,

where $t_{\mathrm{E}}=\theta_{\mathrm{E}} / \mu$, we find the effective proper motion as

$$
\begin{aligned}
\mu & =(4.4 \pm 0.8) \mu \text { as d}^{-1} \\
& =(1.6 \pm 0.3){\text { mas } \mathrm{yr}^{-1}} .
\end{aligned}
$$

With $\pi_{\mathrm{E}}=\pi_{\mathrm{LS}} / \theta_{\mathrm{E}}$ and $\theta_{\mathrm{E}}$, we find

$\pi_{\mathrm{LS}}=(0.48 \pm 0.09)$ mas, 

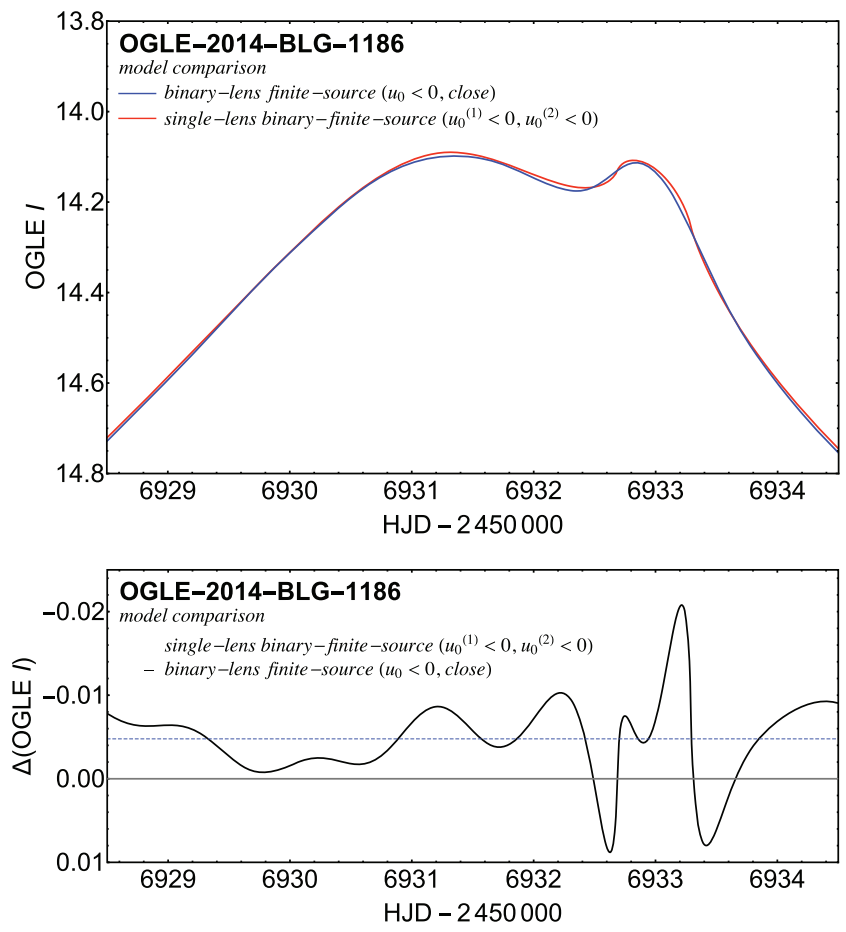

Figure 14. Difference between light curves for the binary-lens finite-source models and the single-lens binary-finite-source models expressed in OGLE $I$ magnitudes. While the photometric uncertainties of peak OGLE data are large in comparison, models adjust to other data with slightly different baseline magnitudes and blend ratios, corresponding to an average shift of about $5 \mathrm{mmag}$ over the peak (indicated by the blue dashed line). The largest difference in shape occurs around the secondary peak during epochs over which no data were acquired.

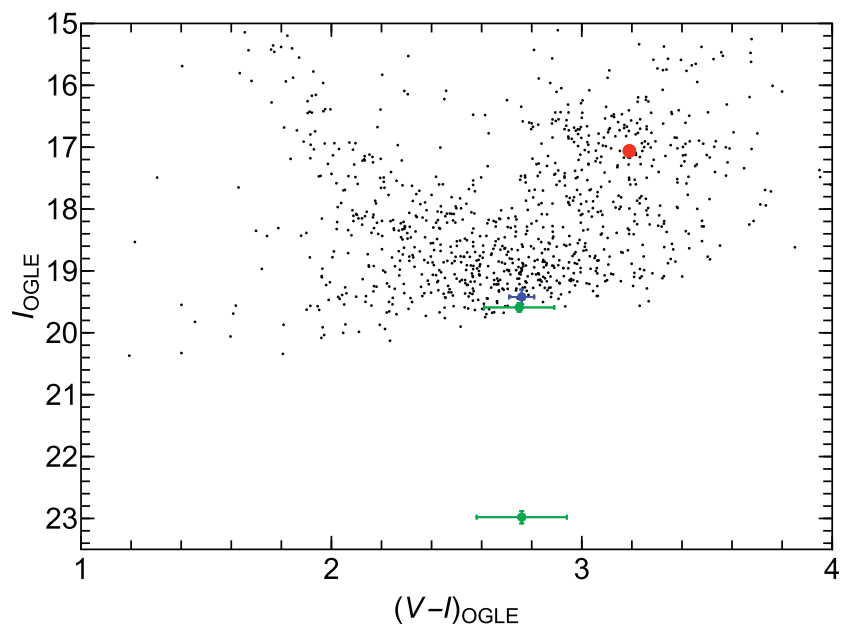

Figure 15. Instrumental CMD for the OGLE field containing the microlensing event OGLE-2014-BLG-1186, marking the location of the centroid of the giant clump (red), as well as the source star(s) for our binary-lens models (blue) or our binary-source models (green).

so that with $D_{\mathrm{S}}$ as given by equation (41), or equivalently

$\pi_{\mathrm{S}}=(0.12 \pm 0.03)$ mas,

one obtains

$\pi_{\mathrm{L}}=(0.60 \pm 0.09)$ mas, equivalent to

$D_{\mathrm{L}}=(1.7 \pm 0.3) \mathrm{kpc}$.

The effective proper motion then implies an effective perpendicular lens velocity $v_{\perp}=D_{\mathrm{L}} \mu$ of

$v_{\perp}=(13 \pm 3) \mathrm{km} \mathrm{s}^{-1}$.

We moreover find the Einstein radius $r_{\mathrm{E}}=D_{\mathrm{L}} \theta_{\mathrm{E}}$, evaluating to

$r_{\mathrm{E}}=(2.0 \pm 0.5) \mathrm{au}$,

and with the binary separation parameters for the close or wide binary case,

$d^{(\mathrm{c})}=0.718 \pm 0.009$,

$d^{(\mathrm{w})}=1.403 \pm 0.014$,

the projected separation at epoch $t_{0}$ becomes

$r_{0, \perp}^{(\mathrm{c})}=(1.4 \pm 0.3) \mathrm{au}$,

$r_{0, \perp}^{(\mathrm{w})}=(2.7 \pm 0.6) \mathrm{au}$.

Consequently, we can estimate the minimal orbital period

$P_{\min }=2 \pi \sqrt{\frac{r_{0, \perp}^{3}}{8 G M}}$

as

$P_{\min }^{(\mathrm{c})}=(1.0 \pm 0.4) \mathrm{yr}$,

$P_{\min }^{(\mathrm{w})}=(2.7 \pm 1.0) \mathrm{yr}$.

The inferred properties and the underlying collated model parameters are comprehensively listed in Table 7.

Kiraga \& Paczyński (1994) originally suggested that $\sim 60$ per cent of all Galactic bulge microlensing events would be caused by bulge stars and $\sim 40$ percent by disc stars, with large uncertainties due to the simplicity of the adopted models and the uncertainty of their model parameters. As noted by Penny, Henderson \& Clanton (2016), it turned out that planet detections reported from observed microlensing events show a strong preference for nearby stars, suggesting that the Galactic bulge stars might be devoid of planets as compared to the Galactic disc stars. The small lens distance $D_{\mathrm{L}} \sim 1.7 \mathrm{kpc}$ further supports this, pointing to a lens star in the disc rather than the bulge. Moreover, this is even substantially less than the average distance of a disc lens star. However, the fact that the Galactic disc is structured into spiral arms, specifically favouring certain ranges of lens distances along the line of sight, should be taken into account. In fact, it is a key goal of observations of microlensing events with the Spitzer space telescope (Calchi Novati et al. 2015; Zhu et al. 2017) to shed light on the distance distribution of microlensing events by combining these with groundbased photometry and thereby measuring the microlensing parallax parameter $\pi_{\mathrm{E}}$. The event time-scale of $t_{\mathrm{E}} \sim 300 \mathrm{~d}$ is much larger than a median of $t_{\mathrm{E}} \sim 20 \mathrm{~d}$ (e.g. Dominik 1998b) expected with best guesses of the stellar mass function (Chabrier 2003). A rather low effective transverse velocity should therefore be expected, and given the large width of the velocity distribution, substantial deviations from the average are within reason. 
Table 7. Overview of collated model parameters and arising physical properties of the lens and source systems.

\begin{tabular}{|c|c|c|}
\hline & Lens binary & Source binary \\
\hline \multicolumn{3}{|l|}{ Collated model parameters } \\
\hline Microlensing parallax parameter & $\pi_{\mathrm{E}}=0.41 \pm 0.01$ & $\pi_{\mathrm{E}}=0.39 \pm 0.01$ \\
\hline Source size parameter & $\rho_{\star}=(10.4 \pm 1.6) \times 10^{-4}$ & $\begin{array}{l}\rho_{\star}^{(1)}=(7.6 \pm 0.5) \times 10^{-3} \\
\rho_{\star}^{(2)}=(1.6 \pm 0.1) \times 10^{-3}\end{array}$ \\
\hline Mass ratio & $q=(3.9 \pm 0.3) \times 10^{-4}$ & - \\
\hline Luminosity offset ratio & - & $\omega_{I}=0.042 \pm 0.003$ \\
\hline Event time-scale & $t_{\mathrm{E}}=(264 \pm 17) \mathrm{d}$ & $t_{\mathrm{E}}=(309 \pm 19) \mathrm{d}$ \\
\hline Binary separation parameter & $\begin{array}{l}d^{(\mathrm{c})}=0.718 \pm 0.009 \\
d^{(\mathrm{w})}=1.403 \pm 0.014\end{array}$ & $\begin{array}{c}\lambda^{\text {cis }}=0.0087 \pm 0.0008 \\
\lambda^{\text {trans }}=0.0107 \pm 0.0009\end{array}$ \\
\hline $\begin{array}{l}\text { Source distance } \\
\text { Baseline magnitude }\end{array}$ & \multicolumn{2}{|c|}{$\begin{array}{c}D_{\mathrm{S}}=(8.5 \pm 2.0) \mathrm{kpc} \\
\quad I_{\text {base }}^{\text {OGLE }}=19.04\end{array}$} \\
\hline \multicolumn{3}{|l|}{ Lens star (system) } \\
\hline Mass of star & $M_{1}=(0.35 \pm 0.06) \mathrm{M}_{\odot}$ & $M=(0.046 \pm 0.007) \mathrm{M}_{\odot}$ \\
\hline Mass of planet & $M_{2}=(45 \pm 9) \mathrm{M}_{\oplus}$ & - \\
\hline Angular Einstein radius & $\theta_{\mathrm{E}}=(1.2 \pm 0.2) \mathrm{mas}$ & $\theta_{\mathrm{E}}=(0.15 \pm 0.02) \mathrm{mas}$ \\
\hline Effective proper motion & $\begin{array}{l}\mu=(4.4 \pm 0.8) \mu \text { as d }^{-1} \\
=(1.6 \pm 0.3) \mathrm{mas} \mathrm{yr}^{-1}\end{array}$ & $\begin{array}{c}\mu=(0.47 \pm 0.08) \mu^{2} \text { as d }^{-1} \\
=(0.17 \pm 0.03) \mathrm{mas} \mathrm{yr}^{-1}\end{array}$ \\
\hline Lens-source parallax & $\pi_{\mathrm{LS}}=(0.48 \pm 0.09) \mathrm{mas}$ & $\pi_{\mathrm{LS}}=(0.057 \pm 0.009) \mathrm{mas}$ \\
\hline Lens distance & $D_{\mathrm{L}}=(1.7 \pm 0.3) \mathrm{kpc}$ & $D_{\mathrm{L}}=(5.7 \pm 0.9) \mathrm{kpc}$ \\
\hline Effective lens velocity & $v_{\perp}=(13 \pm 3) \mathrm{km} \mathrm{s}^{-1}$ & $v_{\perp}=(4.7 \pm 1.1) \mathrm{km} \mathrm{s}^{-1}$ \\
\hline Einstein radius & $r_{\mathrm{E}}=(2.0 \pm 0.5) \mathrm{au}$ & $r_{\mathrm{E}}=(0.84 \pm 0.19) \mathrm{au}$ \\
\hline Current projected separation & $r_{0, \perp}^{(\mathrm{c})}=(1.4 \pm 0.3) \mathrm{au}$ & - \\
\hline & $r_{0, \perp}^{(\mathrm{w})}=(2.7 \pm 0.6) \mathrm{au}$ & - \\
\hline Minimal orbital period & $P_{\min }^{(c)}=(1.0 \pm 0.4) \mathrm{yr}$ & - \\
\hline & $P_{\min }^{(\mathrm{w})}=(2.7 \pm 1.0) \mathrm{yr}$ & - \\
\hline \multicolumn{3}{|l|}{ Source star (system)/microlensing target } \\
\hline Right ascension (J2000) & \multicolumn{2}{|c|}{$\mathrm{RA}=17^{\mathrm{h}} \cdot 41^{\mathrm{m}} \cdot 59.63$} \\
\hline Declination (J2000) & \multicolumn{2}{|c|}{ Dec. $=-34.17^{\prime} \cdot 18^{\prime \prime} .1$} \\
\hline De-reddened red clump colour/mag & \multicolumn{2}{|c|}{$(V-I, I)_{\mathrm{RC}, 0}=(1.06,14.62)$} \\
\hline Red clump colour/mag & \multicolumn{2}{|c|}{$(V-I, I)_{\mathrm{RC}}=(3.19 \pm 0.01,17.06 \pm 0.02)$} \\
\hline Source colour/mag & $(V-I, I)=(2.76 \pm 0.05,19.42 \pm 0.12)$ & $(V-I, I)=(2.75 \pm 0.05,19.54 \pm 0.06)$ \\
\hline De-reddened source colour/mag & $(V-I, I)_{0}=(0.63 \pm 0.05,16.98 \pm 0.12)$ & $(V-I, I)_{0}=(0.62 \pm 0.05,17.10 \pm 0.07)$ \\
\hline De-reddened source colour/mag (1) & - & $(V-I, I)_{0}^{(1)}=(0.62 \pm 0.14,17.15 \pm 0.07)$ \\
\hline De-reddened source colour/mag (2) & - & $(V-I, I)_{0}^{(2)}=(0.63 \pm 0.18,20.54 \pm 0.10)$ \\
\hline Type of source & F V, G IV & F V, G IV / G VI \\
\hline Angular radius of source & $\theta_{\star}=(1.21 \pm 0.11) \mu \mathrm{as}$ & $\begin{array}{l}\theta_{\star}^{(1)}=(1.11 \pm 0.16) \mu \mathrm{as} \\
\theta_{\star}^{(2)}=(0.23 \pm 0.04) \mu \mathrm{as}\end{array}$ \\
\hline Physical radius of source & $R_{\star}=(2.2 \pm 0.6) \mathrm{R}_{\odot}$ & $\begin{array}{c}R_{\star}^{(1)}=(2.0 \pm 0.6) \mathrm{R}_{\odot} \\
R_{\star}^{(2)}=(0.43 \pm 0.13) \mathrm{R}_{\odot}\end{array}$ \\
\hline Angular separation of constituents & $\begin{array}{l}- \\
-\end{array}$ & $\begin{array}{l}\beta^{\text {cis }}=(1.3 \pm 0.2) \mu \mathrm{as} \\
\beta^{\text {trans }}=(1.6 \pm 0.3) \mu \mathrm{as}\end{array}$ \\
\hline Current projected separation & - & $\begin{array}{c}\rho_{\perp}^{\text {cis }}=(0.011 \pm 0.003) \text { au } \\
\rho^{\text {trans }}=(0.013 \pm 0.004) \text { au }\end{array}$ \\
\hline Minimal orbital period & $\begin{array}{l}- \\
-\end{array}$ & $\begin{array}{l}P_{\mathrm{S}, \min }^{\mathrm{cis}}=(0.10 \pm 0.05) \mathrm{d} \\
P_{\mathrm{S}, \min }^{\text {trans }}=(0.14 \pm 0.06) \mathrm{d}\end{array}$ \\
\hline
\end{tabular}

\subsection{Source binary}

With the binary-source models and the binary-lens models having similar blend ratios (within the uncertainties), we find $(V-I, I)=$ $(2.75 \pm 0.05,19.54 \pm 0.06)$ for the combined light of the two source stars. A calibration using the position of the red clump then gives $(V-I, I)_{0}=(0.62 \pm 0.05,17.10 \pm 0.07)$. With a luminosity offset ratio $\omega_{I}=0.042 \pm 0.003$, one finds immediately $I_{0}^{(1)}=$ $17.15 \pm 0.07$ and $I_{0}^{(2)}=20.54 \pm 0.10$. Moreover, the ratio between the angular radii is given by $\rho_{\star}^{(1)} / \rho_{\star}^{(2)}=4.8 \pm 0.4$. Strikingly, the model parameters suggest the ratio between the $I$-band luminosities being roughly the square of the ratio between the angular radii. This implies that the two stars have similar colours. Explicitly, one finds with equation (39), neglecting the quadratic term,

$$
\begin{aligned}
\lg \frac{\theta_{\star}^{(1)}}{\theta_{\star}^{(2)}}= & 0.4895\left[(V-I)_{0}^{(1)}-(V-I)_{0}^{(2)}\right] \\
& -0.2\left(I_{0}^{(1)}-I_{0}^{(2)}\right),
\end{aligned}
$$


leading to $(V-I)_{0}^{(1)}-(V-I)_{0}^{(2)}=0.00 \pm 0.09$ with the estimated values, and consequently to

$$
\begin{gathered}
(V-I, I)_{0}^{(1)}=(0.62 \pm 0.14,17.15 \pm 0.07), \\
V-I, I)_{0}^{(2)}=(0.63 \pm 0.18,20.54 \pm 0.10) .
\end{gathered}
$$

With equation (39), we then obtain the individual angular source radii as ${ }^{5}$

$\theta_{\star}^{(1)}=(1.11 \pm 0.16) \mu \mathrm{as}$,

$\theta_{\star}^{(2)}=(0.23 \pm 0.04) \mu$ as.

With a source distance of $D_{\mathrm{S}}=(8.5 \pm 2.0) \mathrm{kpc}$, these correspond to physical radii

$R_{\star}^{(1)}=(2.0 \pm 0.6) R_{\odot}$,

$R_{\star}^{(2)}=(0.43 \pm 0.13) R_{\odot}$.

The brighter source therefore appears compatible with an F V or G IV star, while the fainter source appears compatible with a G VI star.

Given that our models provide the source size parameters $\rho_{\star}^{(1)}=$ $\theta_{\star}^{(1)} / \theta_{\mathrm{E}}$ and $\rho_{\star}^{(2)}=\theta_{\star}^{(2)} / \theta_{\mathrm{E}}$, where

$\rho_{\star}^{(1)}=(7.6 \pm 0.5) \times 10^{-3}$,

$\rho_{\star}^{(2)}=(1.6 \pm 0.1) \times 10^{-3}$,

the angular Einstein radius is estimated to be

$\theta_{\mathrm{E}}=(0.15 \pm 0.02)$ mas

We find that the angular separation between the source stars is close to the sum of their radii, i.e.

$$
\begin{aligned}
\lambda^{\text {cis }}-\left(\rho_{\star}^{(1)}+\rho_{\star}^{(2)}\right) & =-0.0005 \pm 0.0010, \\
\lambda^{\text {trans }}-\left(\rho_{\star}^{(1)}+\rho_{\star}^{(2)}\right) & =0.0015 \pm 0.0010,
\end{aligned}
$$

with $\lambda^{\text {cis/trans }}$ given by equation (38), which suggests that the source could be a (partially) eclipsing binary, but the two stars could also miss each other. We find an angular separation $\lambda \theta_{\mathrm{E}}$ of

$$
\begin{aligned}
\beta^{\text {cis }} & =(1.3 \pm 0.2) \mu \mathrm{as}, \\
\beta^{\text {trans }} & =(1.6 \pm 0.3) \mu \mathrm{as}
\end{aligned}
$$

and a separation perpendicular to the line of sight $\rho_{\perp}=\lambda D_{\mathrm{S}} \theta_{\mathrm{E}}$ of

$$
\begin{aligned}
\rho_{\perp}^{\text {cis }} & =(0.011 \pm 0.003) \mathrm{AU}, \\
\rho_{\perp}^{\text {trans }} & =(0.013 \pm 0.004) \mathrm{AU} .
\end{aligned}
$$

From the derived stellar types (G IV, F V / G VI), we broadly estimate the masses of the source stars as

$$
\begin{gathered}
M^{(1)}=(1.4 \pm 0.2) \mathrm{M}_{\odot}, \\
M^{(2)}=(0.7 \pm 0.1) \mathrm{M}_{\odot},
\end{gathered}
$$

leading to a total mass $M_{\mathrm{S}}=(2.1 \pm 0.2) \mathrm{M}_{\odot}$. We therefore obtain a minimal orbital period

$$
P_{\mathrm{S}, \min }=2 \pi \sqrt{\frac{\rho_{\perp}^{3}}{8 G M_{\mathrm{S}}}},
$$

${ }^{5}$ In fact, we directly find $\theta_{\star}^{(2)}=\theta_{\star}^{(1)}\left(\rho_{\star}^{(2)} / \rho_{\star}^{(1)}\right)$. evaluating to

$P_{\mathrm{S}, \text { min }}^{\mathrm{cis}}=(0.10 \pm 0.05) \mathrm{d}$,
$P_{\mathrm{S}, \text { min }}^{\text {trans }}=(0.14 \pm 0.06) \mathrm{d}$.

For the parallax parameter $\pi_{\mathrm{E}}=\pi_{\mathrm{LS}} / \theta_{\mathrm{E}}$, the models give

$\pi_{\mathrm{E}}=0.39 \pm 0.01$,

so that with equation (45), one obtains the mass of the lens as

$M=(0.046 \pm 0.007) M_{\odot}$,

compatible with a brown dwarf. Moreover, with

$\pi_{\mathrm{LS}}=(0.057 \pm 0.009) \mathrm{mas}$

and $\pi_{\mathrm{S}}$ as given by equation (53), we find

$\pi_{\mathrm{L}}=(0.17 \pm 0.03)$ mas,

equivalent to

$D_{\mathrm{L}}=(5.7 \pm 0.9) \mathrm{kpc}$.

The Einstein radius $r_{\mathrm{E}}=D_{\mathrm{L}} \theta_{\mathrm{E}}$ therefore becomes

$r_{\mathrm{E}}=(0.84 \pm 0.19) \mathrm{AU}$,

and with the event time-scale

$t_{\mathrm{E}}=(309 \pm 19) \mathrm{d}$,

defined as $\theta_{\mathrm{E}} / \mu$, we obtain the proper motion

$$
\begin{aligned}
\mu & =(0.47 \pm 0.08) \mu \text { as } \mathrm{d}^{-1} \\
& =(0.17 \pm 0.03) \mathrm{mas} \mathrm{yr}^{-1},
\end{aligned}
$$

so that the effective perpendicular lens velocity $v_{\perp}=D_{\mathrm{L}} \mu$ reads

$v_{\perp}=(4.7 \pm 1.1) \mathrm{km} \mathrm{s}^{-1}$.

\subsection{Lens binary or source binary?}

Not only do the acquired photometric data fail to provide sufficient evidence for distinguishing between our binary-lens and binarysource models, but moreover neither of these alternatives lead to an obviously implausible physical nature of the lens or source system, taking into account that the event time-scale of $t_{\mathrm{E}}$ makes the event unusual.

Unfortunately, we missed out on the opportunity to obtain multiband photometry over the peak, but even if we had done so, the discrimination power would have been limited, given that the binary-source models are compatible with the absence of significant colour effects. However, a positive detection of colour differences in the light curve over the peak could have ruled out the binary-lens interpretation.

While orbital motion provides further freedom for both the binary-lens and the binary-source models (Dominik 1998a), a substantial difference lies in the fact that plausible orbital periods are of the order of years for the binary-lens models, but of the order of days for the binary-source models. This means that the flexibility of binary-lens models over the peak is pretty much exhausted, whereas orbital motion can significantly affect the photometric light curve for binary-source models over the peak. However, if we are not certain that the remaining residuals are not due to low-amplitude systematics, we are running the risk that further model tuning would correspond to modelling noise. Moreover, the large number of additional model parameters for fixing a small discrepancy is likely to result in severe ambiguities in an intricate parameter space. 
Despite the caveat that the ratios between the angular source radii and the luminosities of the source stars obtained for the binarysource models might not be somewhat mis-estimated due to orbital motion being mistaken for a contribution to source size, they are remarkably consistent, while one could have easily ended up with implausible properties of the constituents of the source binary, not matching any populated regions of the CMD. This provides some support for the credibility of the binary-source interpretation. It is also interesting that the binary-source model parameters suggest that the source might be a (partially) eclipsing binary. We would definitely know that the source is a binary if (partial) eclipses were found in photometric data. Without (partial) eclipses, there will not be periodicities in the light curve, given that it is well explained by a point-source point-lens model outside the peak region.

The putative binary-source system would also be likely to show a significant variation in radial velocity, detectable in wavelength shifts of characteristic spectral lines (most prominently the Balmer lines as well as the CaII $\mathrm{H}$ and $\mathrm{K}$ lines for $\mathrm{G}$ stars). ${ }^{6}$ For low eccentricity, the orbital velocity is

$v \sim \sqrt{\frac{G M}{a}} \leq \sqrt{\frac{G M}{\rho_{\perp}}}=(390 \pm 70) \mathrm{km} \mathrm{s}^{-1}$,

leading to a velocity of the brighter (and more massive) component of

$v^{(1)}=\frac{M^{(2)}}{M^{(1)}+M^{(2)}} v \lesssim(130 \pm 30) \mathrm{km} \mathrm{s}^{-1}$.

However, we only inferred a lower limit to the semimajor axis and moreover do not know the orientation of the orbit, so that the radial component of the velocity could be smaller. The faintness of the target $(I \sim 19.5)$ is challenging, but spectral variations could already become visible for a modest resolving power $R=\lambda / \Delta \lambda \gtrsim 2500$.

The binary-source and binary-lens models also differ in the nature of the observed blended light, despite the fact that its amount does not differ significantly, given that in both cases the model needs to match the off-peak photometric data which is not affected by binarity. For the binary-source models, the mass of the lens star is suggestive of a brown dwarf, so that the blended light would presumably arise from another star rather than the lens itself. For the binary-lens models, this looks different. Assuming that the lens star of $M \sim 0.4 M_{\star}$ is an M dwarf (M2 V), it would have approximately $M_{V, \mathrm{~L}}=10.2$ and $(V-I)_{\mathrm{L}}=2.16$, i.e. $M_{I, \mathrm{~L}}=8.04$. For the source star, we found $I=16.98 \pm 0.12$. The source distance $D_{\mathrm{S}}=(8.5 \pm 2.0) \mathrm{kpc}$ corresponds to a distance modulus $m-M=$ $14.6 \pm 0.5$, so that $M_{I}=2.3 \pm 0.5$. Moreover, for a lens distance $D_{\mathrm{L}}=(1.7 \pm 0.3) \mathrm{kpc}$, we find $(m-M)_{\mathrm{L}}=11.1 \pm 0.3$, resulting in a relative distance modulus $\Delta(m-M)=3.5 \pm 0.6$. This gives

$\Delta I=M_{I}-M_{I, \mathrm{~L}}+\Delta(m-M)=-2.2 \pm 0.8$,

suggesting that the lens star is fainter than the source star before considering extinction. A blend ratio

$g_{I}=0.41 \pm 0.13$

provides the constraint

$(\Delta I)_{0} \leq 2.5 \lg g_{I}=-1.0 \pm 0.3$

which means that the extinction needs to be

$(\Delta I)_{0}-\Delta I<1.2 \pm 0.9$,

${ }^{6}$ We would like to thank the anonymous reviewer for pointing to this. which appears to be compatible with an average $A_{I}=1.96$ towards the direction of the observed target (Nataf et al. 2013), and some extinction caused by dust between the observer and the lens star. Hence, the lens star is not too bright and might be the main contributor to the blended light. Observing a star compatible with the predicted brightness of the lens star for our binary-lens models that furthermore separates from the source star at a proper motion $\mu=(1.6 \pm 0.3)$ mas $\mathrm{yr}^{-1}$ would give strong support to the binarylens interpretation and constitute evidence against the binary-source interpretation, which has a much fainter lens with a much smaller

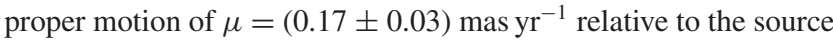
star.

\section{CONCLUSIONS}

The power of inferred planet population statistics from gravitational microlensing campaigns greatly increases with the ability to distinguish low-amplitude signals from the noise floor of photometric data.

Separating model parameters and subsets of data has been demonstrated to be a generic and powerful approach for characterizing localised effects in photometric light curves. In particular, this allows us to build effective models of the photometric noise on data that do not contain the putative signal under investigation, and thereby enables a meaningful probabilistic assessment of the significance of such a signal under the assumption that the data for epochs not covering the signal are reasonably well understood. Hence, signals of planets that are otherwise missed become detectable.

While we laid the groundwork for a detailed assessment of the feasibility of potential alternative model interpretations of the observed data, it turned out that for the concrete case of the microlensing event OGLE-2014-BLG-1186, we can straightforwardly rule out any binary-lens alternatives to the four configurations presented. Rather than claiming that our models are the right ones because no viable alternatives have been found, an analysis of the underlying mathematical properties of potential solutions that can provide matching morphologies enabled us to restrict all viable alternatives within the adopted model framework to a small finite number of prototypes, similar to what was suggested by Liebig et al. (2015), which then either turned out to lead to a match to the data that cannot be improved, or an obvious mismatch. However, we can only check the adopted model framework for plausibility and consistency, whereas it is fundamentally impossible to rule out the existence of further plausible interpretations beyond the adopted model framework, given that our knowledge will always remain limited and incomplete.

In fact, we experienced that close binary source stars pose a challenge for claiming the detection of planets by microlensing in events where the source trajectory passes close to the central caustic near the lens star hosting the planet (Griest \& Safizadeh 1998). This is different from the ambiguity between binary-lens and binary-source interpretations discussed by Gaudi (1998), which relates to planetary signatures arising from approaching planetary caustics. We note that while in this case a small luminosity offset ratio $\omega \lesssim 10^{-2}$ is required, such a restriction does not hold for the type of ambiguity that we encountered. Close binary-source models come with a large number of degrees of freedom, involving two source size parameters as well as parameters that describe the orbital motion, which is likely to significantly affect the light curve over the peak due to orbital periods of the order of days. Binarysource interpretations must not be discarded prematurely on the 
basis of comparing binary-lens models with static binary-pointsource models.

In order to resolve such ambiguities, uninterrupted high-cadence multiband photometric observations over the peak would be useful. Simultaneous or quasi-simultaneous observations with different bandpass filters cannot only measure chromaticity, but moreover increase the statistical significance of signals due to correlations (Dominik \& Hirshfeld 1996; Street et al. 2015). Source binarity could also be indicated by means of spectra taken at different event phases, given the differential magnification between the two source stars or the Doppler shift of spectral lines due to a likely large radial velocity for close binaries. Moreover, the astrometric signature of binary-lens and binary-source events with similar photometric signature is substantially different (Han 2001; Han \& Lee 2002; Han 2002). Calchi Novati et al. (2018) also recently discussed a case of binary-lens versus binary-source ambiguity for an event that shows an anomaly signature both from ground- and space-based photometric observations, providing complementary information due to the different lines of sight.

We finally note that gravitational microlensing events such as OGLE-2014-BLG-1186 for which both the source size parameter $\rho_{\star}$ and the parallax parameter $\pi_{\mathrm{E}}$ can be reliably measured provide a valuable sample for testing models that describe the mass distribution and kinematics of the Milky Way, given that with an estimate of the angular size $\theta_{\star}$ of the source star from a CMD, one directly obtains the mass $M$ of the lens system (as well as the individual masses of its constituents), its distance $D_{\mathrm{L}}$ from the observer, as well as the effective proper motion $\mu$.

\section{ACKNOWLEDGEMENTS}

This publication was made possible by grants NPRP-X-019-1-006 and NPRP-09-476-1-78 from the Qatar National Research Fund (a member of Qatar Foundation). GD acknowledges Regione Campania for support from POR-FSE Campania 2014-2020. MPGH acknowledges support from the Villum Foundation. Work by C.H. was supported by the grant (2017R1A4A1015178) of National Research Foundation of Korea. This work makes use of observations from the LCOGT network, which includes three 'SUPAscopes' owned by the University of St Andrews. The RoboNet programme is an LCOGT Key Project using time allocations from the University of St Andrews, LCOGT and the University of Heidelberg together with time on the Liverpool Telescope through the Science and Technology Facilities Council (STFC), UK. This research has made use of the LCOGT Archive, which is operated by the California Institute of Technology, under contract with the LCO. OGLE Team thanks Profs. M. Kubiak and G. Pietrzyński, former members of the OGLE team, for their contribution to the collection of the OGLE photometric data over the past years. The OGLE project has received funding from the National Science Centre, Poland, grant MAESTRO 2014/14/A/ST9/00121 to AU. LM acknowledges support from the Italian Ministry of Instruction, University and Research (MIUR) through FFABR 2017 fund. LM acknowledges support from the University of Rome Tor Vergata through 'Mission: Sustainability 2016' fund. KH acknowledges support from STFC grant ST/R000824/1.

\section{REFERENCES}

Alard C., 2000, A\&AS, 144, 363

Alard C., Lupton R. H., 1998, ApJ, 503, 325

Albrow M. D. et al., 2000, ApJ, 534, 894
Anderson T. W., Darling D. A., 1952, Ann. Math. Statist, 23, 193

An J. H. et al., 2002, ApJ, 572, 521

Bachelet E. et al., 2015, ApJ, 812, 136

Bachelet E., Norbury M., Bozza V., Street R., 2017, AJ, 154, 203

Beaulieu J.-P. et al., 2006, Nature, 439, 437

Bensby T. et al., 2011, A\&A, 533, A134

Bond I. A. et al., 2004, ApJ, 606, L155

Bozza V. et al., 2012, MNRAS, 424, 902

Bozza V., 2010, MNRAS, 408, 2188

Bramich D. M., 2008, MNRAS, 386, L77

Bramich D. M., Freudling W., 2012, MNRAS, 424, 1584

Calchi Novati S. et al., 2015, ApJ, 804, 20

Calchi Novati S. et al., 2018, AJ, 155, 261

Chabrier G., 2003, PASP, 115, 763

Dominik M. et al., 2002, Planet. Space Sci., 50, 299

Dominik M. et al., 2007, MNRAS, 380, 792

Dominik M. et al., 2008a, in Sun Y.-S., Ferraz-Mello S., Zhou J.-L., eds, IAU Symposium Vol. 249, Exoplanets: Detection, Formation and Dynamics, Cambridge University Press, Cambridge, England, p. 35

Dominik M. et al., 2008b, Astron. Nachr., 329, 248

Dominik M. et al., 2010, Astron. Nachr., 331, 671

Dominik M., 1993, Diploma thesis, Universität Dortmund

Dominik M., 1998a, A\&A, 329, 361

Dominik M., 1998b, A\&A, 330, 963

Dominik M., 1998c, A\&A, 333, L79

Dominik M., 1999, A\&A, 349, 108

Dominik M., Hirshfeld A. C., 1996, A\&A, 313, 841

Dong S. et al., 2009, ApJ, 698, 1826

Einstein A., 1915, Sitzungsber. preuss. Akad. Wiss., 47, 831

Einstein A., 1936, Science, 84, 506

Erdl H., Schneider P., 1993, A\&A, 268, 453

Evans D. F. et al., 2016, A\&A, 589, A58

Gaudi B. S. et al., 2008, Science, 319, 927

Gaudi B. S., 1998, ApJ, 506, 533

Gaudi B. S., Sackett P. D., 2000, ApJ, 528, 56

Gould A., 2004, ApJ, 606, 319

Gould A., Gaucherel C., 1997, ApJ, 477, 580

Gould A., Loeb A., 1992, ApJ, 396, 104

Griest K., Hu W., 1992, ApJ, 397, 362

Griest K., Safizadeh N., 1998, ApJ, 500, 37

Han C., 2001, MNRAS, 325, 1281

Han C., 2002, ApJ, 564, 1015

Han C., Lee C., 2002, MNRAS, 329, 163

Harpsøe K. B. W., Jørgensen U. G., Andersen M. I., Grundahl F., 2012, A\&A, 542, A23

Hoaglin D. C., Mosteller F., Tukey J. W., eds, 1983, Understanding Robust and Exploratory Data Analysis. Wiley, New York

Horne K., Snodgrass C., Tsapras Y., 2009, MNRAS, 396, 2087

Huber P. J., Ronchetti E. M., 2009, Robust statistics, 2nd edn. Wiley, New York

Hundertmark M. et al., 2018, A\&A, 609, A55

James F., Roos M., 1975, Comput. Phys. Commun., 10, 343

Janczak J. et al., 2010, ApJ, 711, 731

Kervella P., Fouqué P., 2008, A\&A, 491, 855

Kiraga M., Paczyński B., 1994, ApJ, 430, L101

Liebig C., D’Ago G., Bozza V., Dominik M., 2015, MNRAS, 450, 1565

Mao S., Paczyński B., 1991, ApJ, 374, L37

Muraki Y. et al., 2011, ApJ, 741, 22

Nataf D. M. et al., 2013, ApJ, 769, 88

Paczyński B., 1986, ApJ, 304, 1

Penny M. T., Henderson C. B., Clanton C., 2016, ApJ, 830, 150

Rhie S. H. et al., 2000, ApJ, 533, 378

Skottfelt J. et al., 2015, A\&A, 574, A54

Skowron J. et al., 2011, ApJ, 738, 87

Skowron J. et al., 2016, AcA, 66, 1

Skowron J., Gould A., 2012, preprint (arXiv:1203.1034)

Street R. A., Fulton B. J., Scholz A., Horne K., Helling C., Juncher D., Lee G., Valenti S., 2015, ApJ, 812, 161 
Sumi T. et al., 2010, ApJ, 710, 1641

Tsapras Y. et al., 2009, Astron. Nachr., 330, 4

Tsapras Y., Horne K., Kane S., Carson R., 2003, MNRAS, 343, 1131

Udalski A. et al., 2005, ApJ, 628, L109

Udalski A., Szymanski M., Kaluzny J., Kubiak M., Mateo M., Krzeminski W., Paczynski B., 1994, AcA, 44, 227

Udalski A., Szymański M. K., Szymański G., 2015, AcA, 65, 1

Witt H. J., Mao S., 1994, ApJ, 430, 505

Witt H. J., Mao S., 1995, ApJ, 447, L105

Woźniak P. R., 2000, AcA, 50, 421

Zhu W. et al., 2017, AJ, 154, 210

${ }^{1}$ Centre for Exoplanet Science, SUPA School of Physics \& Astronomy, University of St Andrews, North Haugh, St Andrews KY16 9SS, $U K$

${ }^{2}$ Las Cumbres Observatory Global Telescope Network, 6740 Cortona Drive, suite 102, Goleta, CA 93117, USA

${ }^{3}$ Qatar Environment and Energy Research Institute (QEERI), HBKU, Qatar Foundation, Doha, Qatar

${ }^{4}$ Dipartimento di Fisica 'E. R. Caianiello', Università di Salerno, Via Giovanni Paolo II 132, I-84084 Fisciano (SA), Italy

${ }^{5}$ Istituto Nazionale di Fisica Nucleare, Sezione di Napoli, Via Cintia, 80126 Napoli, Italy

${ }^{6}$ Department of Physics, Chungbuk National University, Cheongju 28644, Republic of Korea

${ }^{7}$ Niels Bohr Institute \& Centre for Star and Planet Formation, University of Copenhagen, Øster Voldgade 5, DK-1350 Copenhagen K, Denmark

${ }^{8}$ Astronomisches Rechen-Institut, Zentrum für Astronomie der Universität Heidelberg (ZAH), D-69120 Heidelberg, Germany

${ }^{9}$ Warsaw University Observatory, Al. Ujazdowskie 4, PL-00-478 Warszawa,

Poland

${ }^{10}$ New York University Abu Dhabi, Saadiyat Island, Abu Dhabi, PO Box 129188, United Arab Emirates

${ }^{11}$ IPAC, Mail Code 100-22, Caltech, 1200 E. California Blvd., Pasadena, CA 91125, USA

${ }^{12}$ Max Planck Institute for Astronomy, Königstuhl 17, D-69117 Heidelberg,

Germany

${ }^{13}$ International Institute for Advanced Scientific Studies (IIASS), Via G. Pellegrino 19, I-84019 Vietri sul Mare (SA), Italy

${ }^{14}$ INAF - Osservatorio Astronomico di Capodimonte, Salita Moiarello 16, I-80131 Napoli, Italy

${ }^{15}$ Instituto de Astrofísica, Pontficia Universidad Católica de Chile, Av. Vicuña Mackenna 4860, 7820436 Macul, Santiago, Chile
${ }^{16}$ European Southern Observatory, Karl-Schwarzschild-Str. 2, D-85748 Garching bei München, Germany

${ }^{17}$ Physics Department and Tsinghua Centre for Astrophysics, Tsinghua University, Beijing 100084, China

${ }^{18}$ Chungnam National University, Department of Astronomy and Space Science, Daejeon 34134, Republic of Korea

${ }^{19}$ Korea Astronomy \& Space Science Institute, 776 Daedukdae-ro, Yuseonggu, 305-348 Daejeon, Republic of Korea

${ }^{20}$ Space Telescope Science Institute, 3700 San Martin Drive, Baltimore, MD 21218, USA

${ }^{21}$ Dark Cosmology Centre, Niels Bohr Institute, University of Copenhagen, Juliane Maries Vej 30, DK-2100 Copenhagen, Denmark

${ }^{22}$ Department of Physics, University of Rome Tor Vergata, Via della Ricerca Scientifica 1, I-00133 Roma, Italy

${ }^{23}$ INAF - Astrophysical Observatory of Turin, Via Osservatorio 20, I-10025 Pino Torinese, Italy

${ }^{24}$ South African Astronomical Observatory, PO Box 9, Observatory 7935, South Africa

${ }^{25}$ Department of Physics, Sharif University of Technology, PO Box $11155-$ 9161 Tehran, Iran

${ }^{26}$ Centre for Electronic Imaging, Department of Physical Sciences, The Open University, Milton Keynes MK7 6AA, UK

${ }^{27}$ Planetary and Space Science, Department of Physical Sciences, The Open University, Milton Keynes MK7 6AA, UK

${ }^{28}$ Max-Planck-Institut für Sonnensystemforschung, Justus-von-Liebig-Weg 3, D-37077 Göttingen, Germany

${ }^{29}$ Institute for Astronomy, University of Edinburgh, Royal Observatory, Edinburgh EH9 3 HJ, UK

${ }^{30}$ Astrophysics Group, Keele University, Keele, Newcastle ST5 5BG, UK

${ }^{31}$ Astrophysics Research Institute, Liverpool John Moores University, Liverpool CH41 1LD, UK

${ }^{32}$ Institut d'Astrophysique et de Géophysique, Allée du 6 Août 19c, Sart Tilman, 4000 Liège, Belgium

${ }^{33}$ School of Physics and Astronomy, Queen Mary University of London, Mile End Road, London E1 4NS, UK

${ }^{34}$ International Space Science Institute, Hallerstrasse 6, CH-3012 Bern, Switzerland

${ }^{35}$ Department of Physics, University of Warwick, Gibbet Hill Road, Coventry CV4 7AL, UK

${ }^{36}$ Department of Astronomy, Ohio State University, 140 W. 18th Ave., Columbus, OH 43210, USA

This paper has been typeset from a $\mathrm{T}_{\mathrm{E}} \mathrm{X} / \mathrm{L} \mathrm{T}_{\mathrm{E}} \mathrm{X}$ file prepared by the author. 